\title{
Nonlinear dynamics of the viscoelastic Kolmogorov flow
}

\author{
$\begin{array}{llllllllllllllllllll}\text { By } & \text { A. } & \text { B I } & \mathbf{S} & \mathbf{T} & \mathbf{A} & \mathbf{G} & \mathbf{N} & \mathbf{I} & \mathbf{N} & \mathbf{O}^{1}, & \mathbf{G} . & \mathbf{B} & \mathbf{O} & \mathbf{F} & \mathbf{F} & \mathbf{E} & \mathbf{T} & \mathbf{T} & \mathbf{A}^{1},\end{array}$ \\ A. CELA N I ${ }^{2}, \quad$ A. MAZZI N O ${ }^{3,5}, \quad$ A. P ULIA F I T O $\mathbf{O}^{2,3}$ \\ AND M. VERGASSOL A \\ ${ }^{1}$ Dipartimento di Fisica Generale, Università di Torino and Istituto Nazionale di Fisica \\ Nucleare, Sez. di Torino, V. Giuria 1, 10125 Torino, Italy \\ ${ }^{2}$ CNRS, INLN, 1361 Route des Lucioles, 06560 Valbonne, France \\ 3 Dipartimento di Fisica, Università di Genova, Via Dodecaneso 33, 16146 Genova, Italy \\ ${ }^{4}$ CNRS URA 2171, Inst. Pasteur, 25 rue du Dr. Roux, 75724 Paris Cedex 15, France. \\ ${ }^{5}$ Istituto Nazionale di Fisica Nucleare, Sez. di Genova, Via Dodecaneso 33, I-16146 Genova, \\ Italy
}

(Received 5 June 2018)

The weakly nonlinear regime of a viscoelastic Navier-Stokes fluid is investigated. For the purely hydrodynamic case, it is known that large-scale perturbations tend to the minima of a Ginzburg-Landau free-energy functional with a double-well (fourth-order) potential. The dynamics of the relaxation process is ruled by a one-dimensional CahnHilliard equation that dictates the hyperbolic tangent profiles of kink-antikink structures and their mutual interactions. For the viscoelastic case, we found that the dynamics still admits a formulation in terms of a Ginzburg-Landau free-energy functional. For sufficiently small elasticities, the phenomenology is very similar to the purely hydrodynamic case: the free-energy functional is still a fourth-order potential and slightly perturbed kink-antikink structures hold. For sufficiently large elasticities, a critical point sets in: the fourth-order term changes sign and the next-order nonlinearity must be taken into account. Despite the double-well structure of the potential, the one-dimensional nature of the problem makes the dynamics sensitive to the details of the potential. We analysed the interactions among these generalized kink-antikink structures, demonstrating their role in a new, elastic instability. Finally, consequences for the problem of polymer drag reduction are presented.

\section{Introduction}

The derivation of coarse-grained equations of motion, averaging out microscopic degrees of freedom and retaining only those features relevant to the process of interest, is a major goal in many different scientific domains. A first classical example is the dynamics of celestial bodies, the physical problem which motivated the introduction of asymptotic techniques to systematically average over rapidly rotating, angular degrees of freedom. More recently, many interesting phenomena in biological contexts (e.g. related to domain formation in lipid membrane, bilayer fusion, and cooperative motions associated with phase changes) have been found to occur on times and length scales much larger than the typical times and scales where the classical molecular-dynamics methods are applicable Vattulainen \& Karttunen 2005). To reach those larger length-scales, one resorts 
to coarse-grained models that employ effective interaction potentials Karttunen et al. 2004).

Another relevant example of coarse-grained model comes from climatology. The current numerical models for prediction of weather and climate involve general circulation models. They consist of coupled, nonlinear partial differential equations, discretized in space and time for the purpose of numerical simulations. The current generation of supercomputers supports mesh spacing of the order of $200 \mathrm{~km}$ for short-term climate simulations. However, many important physical processes occur on smaller scales (e.g. the cloud cover in the boundary layer) and they significantly affect the large-scale dynamics of resolved fields. A powerful way to incorporate the unresolved dynamics is provided by suitable coarse-grained stochastic models (Khouider et al. 2003).

Finally, in the framework of phase-ordering kinetics, the concept of coarse-grained description plays a crucial role for the order-parameter dynamics. Coarsening is intimately related to the fact that domain growth is a scaling phenomenon: domain patterns at different times differ solely by a global scale factor (see the review by Brav 2002). A suitable coarse-grained description for systems where the order parameter is not conserved (e.g. for anti-ferromagnetic ordering) is provided by the time-dependent Ginzburg-Landau equation. When the order parameter is conserved, as in phase separation, the coarse-grained dynamics is ruled by the Cahn-Hilliard equations (Brav 2002):

$$
\frac{\partial w}{\partial t}=\partial_{x}^{2} \frac{\delta F}{\delta w}
$$

where $w(\mathbf{x}, t)$ is a suitable coarse-grained order-parameter and $F$ a Landau free-energy functional:

$$
F[w]=\int \mathrm{d} \mathbf{x}\left[\frac{\lambda}{2}|\nabla w|^{2}+I(w)\right] .
$$

The potential $I(w)$ typically has a double-well structure, whose minima correspond to two equilibrium states. $\lambda$ is a positive constant related to the distance between the equilibrium states and thus the size of the interface between them.

In fluid mechanics, the Cahn-Hilliard equations (1.1) play a fundamental role in the stability analysis of large-scale perturbations. In a variety of situations, it turns out that the evolution of large-scale perturbations is governed by equation (1.1), with a fourth-order potential $I(w)$ (see Nepomnvashchvi 1976: Sivashinskv 1985; Pedloskv 1987; Manfroi \& Young 1999). The structure of the potential controls the profile and the interactions of the so-called kink-antikink structures observed in snapshots of the flow (She 1987).

In the present paper, we focus our attention on a simple model of viscoelastic flows, the so-called viscoelastic Kolmogorov flow. Its linear stability analysis has been recently investigated by Boffetta et al. (2005a), while the turbulent regime and its massive drag reduction effects have been studied by Boffetta et al. (2005b). Here, we analyse the weakly nonlinear dynamics, intermediate between the linear stage of evolution and the fully turbulent regime.

The starting points of our analysis are three results obtained by Boffetta et al. (2005a) for the linearized stage: i) The most unstable perturbation has a long wavelength (largescale) compared to the period of the basic Kolmogorov flow; ii) Its linear evolution is captured by asymptotic multiscale methods, at least up to moderate elasticities of the flow; iii) The most unstable perturbation is transverse with respect to the basic flow.

Multiscale asymptotic methods can be applied, as in the Newtonian case, to show that the evolution in the presence of polymers obeys a one-dimensional Cahn-Hilliard equation of the form (1.1). The point demonstrated here is that there exists a critical 
value of the elasticity, where the potential $I(w)$ passes from the fourth to the sixth order in the field $w$. This corresponds to a triple critical point. Due to the one-dimensional character of the nonlinear dynamics, the transition strongly impinges on the dynamics of the large-scale perturbation.

Above the critical elasticity, "hydrodynamic" kink-antikink structures are replaced by generalized kinks and anti-kinks and their annihilation processes are shown to be severely slowed down. Moreover, below the critical value of the elasticity, the mechanism of instability is linear and nonlinear terms stabilize the flow. Conversely, above the critical value, we show that a sub-critical, nonlinear mechanism of instability takes place, provided the initial amplitude of the perturbation be sufficiently strong.

The paper is organized as follows. In $\S 2$ and 3 we describe the viscoelastic model considered in the sequel and briefly review the results by Boffetta et al. (2005a) needed here. In $\S$, we use multiscale methods to derive the coarse-grained equations for the

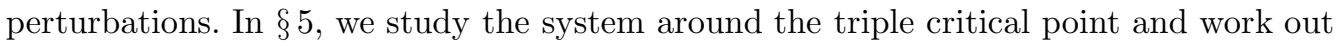
the evolution equations in its neighborhood. In $\S 6$ and 7 we reformulate the asymptotic behaviour of the coarse-grained equations in terms of variational analysis and present the numerical results that corroborate our analytical predictions. Finally, in $\oint 8$ we address the problem of drag reduction and show that, even for the weakly unstable regime considered here, the injection of polymers induces an enhancement of the mean flow amplitude.

\section{The viscoelastic Navier-Stokes equations}

Several models have been introduced (see e.g. Hinch 1977) to describe viscoelastic fluids. A powerful class describes the fluid as non-Newtonian, accounting for the reaction of the polymers onto the flow via an extra-term in the stress tensor. A popular and often employed model within this class is the Oldroyd-B (Oldrovd 1950), which is the one considered in the sequel. We briefly review it here for the sake of completeness.

In the Oldroyd-B model, it is assumed that viscoelastic flows can be treated as a dilute suspension of elastic dumbbells, i.e. identical pairs of microscopic beads connected by Hookean springs. The flow is considered "external" to the molecule, neglecting the effects of the finite size of the polymers on the flow. Furthermore, the polymer concentration is supposed to be uniform and low enough to neglect polymer-polymer interactions.

The reaction of the dumbbells on the fluid is treated at a mean-field level and the study of the dynamics is limited to scales much larger than the inter-polymer distance. The polymer solution is regarded as a continuous medium, whose reaction on the flow is described by an elastic contribution $\boldsymbol{T}$ to the total stress tensor of the fluid. Its value per unit density depends on the free energy of the molecule and the thermal noise as (see e.g. Bird et al. 1987):

$$
\boldsymbol{T}=-n_{p}\langle\boldsymbol{R F}\rangle-n_{p} k_{B} \Theta \mathbf{1},
$$

where $n_{p}$ is the polymer density, $k_{B} \Theta$ is the energy associated with thermal noise, $F_{i}$ is the dumbbell relaxation force and $R_{i}$ its elongation vector. The average is taken over the statistics of the thermal noise. Assuming the force between the beads to be Hookean with dynamical coefficient $K_{0}$, the average in the elastic stress reduces to $\langle\boldsymbol{R} \boldsymbol{F}\rangle=-K_{0}\langle\boldsymbol{R} \boldsymbol{R}\rangle$. The latter is proportional to the conformation tensor $\boldsymbol{\sigma} \equiv\langle\boldsymbol{R} \boldsymbol{R}\rangle / R_{0}^{2}$, where $R_{0}$ denotes the equilibrium spring length. The inclusion of the extra elastic stress term in the NavierStokes equations leads to the following equation for the viscoelastic flow:

$$
\partial_{t} \boldsymbol{v}+(\boldsymbol{v} \cdot \boldsymbol{\partial}) \boldsymbol{v}=-\boldsymbol{\partial} p+\nu \beta \partial^{2} \boldsymbol{v}+\frac{\nu(1-\beta)}{\tau} \boldsymbol{\partial} \cdot(\boldsymbol{\sigma}-\mathbf{1})+\boldsymbol{f} .
$$

Here, $\nu$ is the total kinematic viscosity of the solution, while $\nu \beta$ and $\nu(1-\beta)$ are the sepa- 
rate contributions by the solvent and the polymers, respectively, and we have introduced the dimensionless parameter $\beta=\eta_{s} /\left(n_{p} k_{B} \Theta \tau+\eta_{s}\right), \eta_{s}$ being the dynamic viscosity of the solvent. $\tau$ is a parameter depending on $K_{0}$ and $R_{0}$, representing the typical relaxation time of the polymers. A more precise definition of $\tau$ and $\sigma$ comes in the following. Throughout the paper, it is understood that $(\boldsymbol{\partial} \boldsymbol{v})_{\alpha \beta} \equiv \partial_{\alpha} v_{\beta}$ and $\operatorname{tr}(\boldsymbol{\partial} \boldsymbol{v})=\boldsymbol{\partial} \cdot \boldsymbol{v}=0$.

An equation for the dynamics of the polymer conformation tensor $\boldsymbol{\sigma}$ is needed to close the system of equations. Simple physical reasoning by Bird et al. (1987) gives the following stochastic equation for the separation $\boldsymbol{R}$ between two beads:

$$
\dot{\boldsymbol{R}}=(\boldsymbol{R} \cdot \boldsymbol{\partial}) \boldsymbol{v}-\frac{1}{2 \tau} \boldsymbol{R}+\sqrt{\frac{R_{0}^{2}}{\tau}} \boldsymbol{\xi} .
$$

On the right-hand side, the first term is the stretching/compression term, originating from the spatial variation of the flow experienced by $\boldsymbol{R}$, and the last one, $\boldsymbol{\xi}$, is a white-intime random process mimicking the effect of thermal noise on the polymers. The second is the relaxation due to the force between the beads, proportional to the elongation derivative of the dumbbell free energy $-\partial E / \partial R_{i}=-\partial\left(1 / 2 K_{0} R^{2}\right) / \partial R_{i}$. A quadratic form of the potential, and thus a linear Hookean force, is an approximation valid for moderate polymer elongations. The dynamical coefficient $\tau$ is the same as the one appearing in (2.2) . Considering it constant amounts to assume that the polymers have only one relaxation time. Numerical and theoretical studies point out that this hypothesis is reasonable (Geraschenko et al. 2005). Experiments (see Lumlev 1969; Virk 1975; Nadolink \& Haigh 1995) show that polymers have a spectrum of typical relaxation times, but they also show that interactions with the fluid mostly depend on the largest one, that is the one we are retaining.

Multiplying (2.3) by $\boldsymbol{R}$ and averaging over the statistics of the thermal noise $\boldsymbol{\xi}$, the following evolution equation for the conformation tensor $\boldsymbol{\sigma}=\langle\boldsymbol{R} \boldsymbol{R}\rangle / R_{0}^{2}$ is obtained (see Bird et al. 1987):

$$
\partial_{t} \boldsymbol{\sigma}+(\boldsymbol{v} \cdot \boldsymbol{\partial}) \boldsymbol{\sigma}=(\boldsymbol{\partial} \boldsymbol{v})^{T} \cdot \boldsymbol{\sigma}+\boldsymbol{\sigma} \cdot(\boldsymbol{\partial} \boldsymbol{v})-\frac{1}{\tau}(\boldsymbol{\sigma}-\mathbf{1}) .
$$

Summarizing, the set of equations (2.2) and (2.4) constitutes the Oldroyd-B model that we shall be considering in the sequel.

Our first step in the investigation of the effect of polymers onto the stability of the flow will be to find out the basic equilibrium state. The state will then be perturbed and the resulting equations analysed using multiscale methods.

\subsection{A basic equilibrium state}

Finding analytically the basic equilibrium state for a generic forcing $f$ is a hopeless task already for the Navier-Stokes equations without polymers. The problem is further complicated here by the additional term in (2.2) and the coupling with (2.4).

The problem simplifies for $\boldsymbol{f} \equiv(f(z), 0,0)$, inducing a parallel flow $\boldsymbol{U}=(U(z), 0,0)$, which trivially annihilates the advective nonlinear term in (2.2). A further substantial simplification comes from the viscoelastic version of Squire's theorem (see Appendix A), stating that, for parallel flows, the most unstable perturbations are two-dimensional. We shall therefore restrict to a two-dimensional flow $\left(u_{x}, u_{z}\right)$, without any lack of generality (see also Boffetta et al. 2005a). We further assume $f(z)=F_{0} \cos (z / L)$, producing the well-known Kolmogorov flow (Arnold \& Meshalkin 1960) $U(z) \equiv(V \cos (z / L), 0)$, where $V=F_{0} L^{2} / \nu$. The corresponding conformation tensor at equilibrium is:

$$
\boldsymbol{\sigma}=\left(\begin{array}{cc}
1+2 \tau^{2}\left(\partial_{z} U\right)^{2} & \tau \partial_{z} U \\
\tau \partial_{z} U & 1
\end{array}\right)=\left(\begin{array}{cc}
1+2 \tau^{2} \frac{V^{2}}{L^{2}} \sin ^{2}\left(\frac{z}{L}\right) & -\tau \frac{V}{L} \sin \left(\frac{z}{L}\right) \\
-\tau \frac{V}{L} \sin \left(\frac{z}{L}\right) & 1
\end{array}\right)
$$


This choice also allows to precisely define the Reynolds number of the flow as $R e=V L / \nu$. In this model the elasticity of the polymers is taken into account by the relaxation time $\tau$ only. We can thus introduce an adimensional parameter, the Deborah number $D e=\tau V / L$, to characterize the elastic properties of the flow.

\section{Some previous results on the linear stability analysis}

It has long been known that the Newtonian Kolmogorov flow becomes unstable for Reynolds numbers $R e>\sqrt{2}$ (Meshalkin \& Sinai 1961): the evolution of large-scale perturbations is formally described by an effective diffusive dynamics and instabilities are associated to the loss of positive-definiteness of the eddy-viscosity tensor.

In the presence of polymers, performing a multiscale analysis (Bensoussan et al. 1978; Bavlv et al. 1988) on the linearized Oldroyd-B model, one obtains an explicit expression for the eddy-viscosity tensor, valid for sufficiently low elasticity (Boffetta et al. 2005a). The resulting stability curve in terms of the Reynolds and the Deborah number is reported in figure 1

\subsection{Multiscale analysis}

Substituting $\boldsymbol{v}=\boldsymbol{u}+\boldsymbol{w}$ into (2.22.4), the equations for the perturbation fields read:

$$
\begin{gathered}
\boldsymbol{\partial} \cdot \boldsymbol{w}=0, \\
\partial_{t} \boldsymbol{w}+\boldsymbol{\partial} \cdot(\boldsymbol{u} \boldsymbol{w}+\boldsymbol{w} \boldsymbol{u}+\boldsymbol{w} \boldsymbol{w})=-\boldsymbol{\partial} q+\nu \beta \partial^{2} \boldsymbol{w}+\nu(1-\beta) \tau^{-1} \boldsymbol{\partial} \cdot \boldsymbol{\zeta}, \\
\partial_{t} \boldsymbol{\zeta}+\boldsymbol{\partial} \cdot(\boldsymbol{u} \boldsymbol{\zeta}+\boldsymbol{w} \boldsymbol{\sigma}+\boldsymbol{w} \boldsymbol{\zeta})=(\boldsymbol{\partial u})^{T} \cdot \boldsymbol{\zeta}+(\boldsymbol{\partial w})^{T} \cdot \boldsymbol{\sigma}+(\boldsymbol{\partial} \boldsymbol{w})^{T} \cdot \boldsymbol{\zeta}+ \\
+\boldsymbol{\zeta} \cdot(\boldsymbol{\partial u})+\boldsymbol{\sigma} \cdot(\boldsymbol{\partial} \boldsymbol{w})+\boldsymbol{\zeta} \cdot(\boldsymbol{\partial w})-\tau^{-1} \boldsymbol{\zeta},
\end{gathered}
$$

where $q$ and $\zeta$ are the perturbations associated to the pressure term $p$ and the basic stress tensor $\boldsymbol{\sigma}$. In the linear stability analysis, the nonlinear terms containing the product of two perturbation fields are supposed to be negligible (see Boffetta et al. 2005a).

As in the Newtonian case, it is assumed that the first unstable perturbations have periodicity much larger than that of the basic flow. The validity of this assumption has already been investigated by Boffetta et al. (2005a) and is satisfied in the range of parameters considered here.

In addition to the usual "fast" space/time variables $\boldsymbol{x}, t$, describing the basic flow, multiscale techniques introduce "slow" variables $\tilde{\boldsymbol{x}}=\epsilon \boldsymbol{x}, \tilde{t}=\epsilon^{2} t$, to describe the largescale flow, and prescribe to treat the two sets as independent. This leads to the expansion of the differential operators:

$$
\partial_{i} \rightarrow \partial_{i}+\epsilon \tilde{\partial}_{i}, \quad \partial_{t} \rightarrow \partial_{t}+\epsilon^{2} \tilde{\partial}_{t},
$$

and of the fields:

$$
\begin{aligned}
& \boldsymbol{w}=\boldsymbol{w}^{(0)}(z, t, \tilde{x}, \tilde{z}, \tilde{t})+\epsilon \boldsymbol{w}^{(1)}(z, t, \tilde{x}, \tilde{z}, \tilde{t})+\epsilon^{2} \boldsymbol{w}^{(2)}(z, t, \tilde{x}, \tilde{z}, \tilde{t})+\ldots, \\
& q=q^{(0)}(z, t, \tilde{x}, \tilde{z}, \tilde{t})+\epsilon q^{(1)}(z, t, \tilde{x}, \tilde{z}, \tilde{t})+\epsilon^{2} q^{(2)}(z, t, \tilde{x}, \tilde{z}, \tilde{t})+\ldots \\
& \boldsymbol{\zeta}=\boldsymbol{\zeta}^{(0)}(z, t, \tilde{x}, \tilde{z}, \tilde{t})+\epsilon \boldsymbol{\zeta}^{(1)}(z, t, \tilde{x}, \tilde{z}, \tilde{t})+\epsilon^{2} \boldsymbol{\zeta}^{(2)}(z, t, \tilde{x}, \tilde{z}, \tilde{t})+\ldots
\end{aligned}
$$

All of the functions have the periodicity of the basic flow and are independent of $x$.

Inserting (3.5) into (3.1)-(3.3) and collecting terms of the same order in $\epsilon$, the coarsegrained equation for a large-scale perturbation is obtained as the solvability condition at the order $\epsilon^{2}$. In terms of the stream function $\Psi$, the perturbation evolves according to the non-isotropic diffusion equation:

$$
\tilde{\partial}_{t} \tilde{\Delta} \Psi=\nu_{\alpha \beta} \tilde{\partial}_{\alpha}^{2} \tilde{\partial}_{\beta}^{2} \Psi
$$




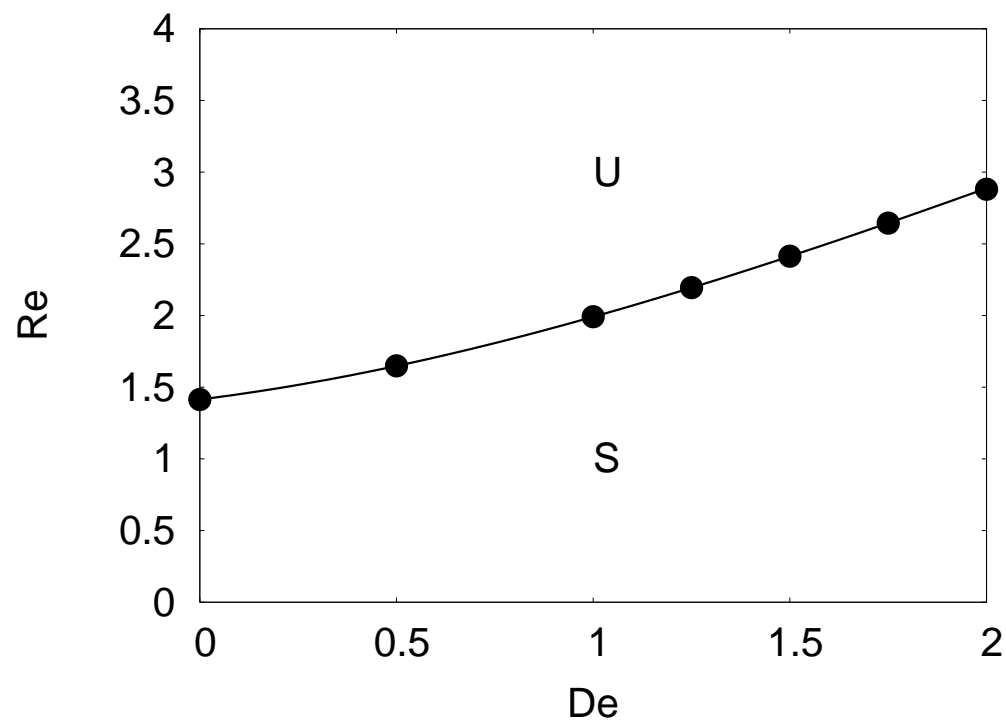

Figure 1 . The linear stability diagram for $\beta=0.769$. Stable and unstable regions are denoted by $\mathrm{S}$ and U, respectively. The bullets represent direct numerical simulations (DNS) of the complete Oldroyd-B system of equations, confirming theoretical predictions for this window of parameters.

where the eddy-viscosity tensor $\nu_{\alpha \beta}$ is not positive-definite for $R e>\sqrt{2}$ (in the absence of polymers). In general, there exist critical values of $R e$ and $D e$ where perturbations start growing in time.

The phase-space $(R e, D e)$ is thus divided in regions where the eddy-viscosity tensor is positive-definite (the system is linearly stable with respect to any small perturbation) and where there exists at least one unstable mode, as shown in figure 1 for low Deborah numbers. The diagram reveals two kinds of instabilities. When the Deborah number is sufficiently low, the flow experiences hydrodynamic-like large-scale transverse instabilities, captured by multiscale analysis. In this region, the critical Reynolds number where the flow becomes unstable, grows with De: polymers stabilize the flow. This has been interpreted by Boffetta et al. (2005a), and will be shown in $\S[$ to be a prelude to the drag reduction effect observed in the turbulent regime.

For high values of the Deborah number (not shown in figure 1) the multiscale analysis predicts the flow to be unstable, even for very low Reynolds numbers. However, numerical simulations show that the assumption of scale separation does not hold and multiscale techniques are not applicable. This region, possibly characterized by purely elastic instabilities, will not be the concern of the present investigation which focuses on $0 \leq D e \leq 2$.

If the amplitude of the large-scale perturbation is strong enough and/or the eddyviscosity is negative, nonlinear effects are important and should be taken into account. These two situations correspond to different scalings of the fields and will be treated separately in the next sections.

\section{Nonlinear dynamics: the standard Cahn-Hilliard equation}

Linear stability analyses, by their very definition, are not able to capture the full-time dynamics of unstable perturbations: as perturbations grow in time, their magnitude becomes large and nonlinearities ought to be taken into account. In the Newtonian case 
(Sivashinskv 1985; She 1987), the lowest-order nonlinearity (third-order) is sufficient to stabilize the linear exponential growth and lead to a steady state. Here, we show how this occurs and generalize it first to the viscoelastic case. The next section will then be devoted to the case where the lowest-order nonlinearity does not stabilize the flow and higher-order nonlinearities become relevant.

A careful analysis of the linear eddy-viscosity tensor $\nu_{\alpha \beta}$ derived in (3.6) ensures that for low enough Deborah numbers the first modes to become linearly unstable are the large-scale transverse ones. For barely unstable flows we may expect that the perturbations involved in the nonlinear dynamics will be confined to these modes. This suggests that the result will be a one-dimensional diffusion equation for the averaged transverse modes, linearly stable for small-scale modes, involving at least one nonlinear term.

Assume now that the initial amplitude of the large-scale perturbation is sufficiently small and the system we consider is in the surroundings of a point of the critical curve. According to (3.6), the average transverse velocity perturbation $\left\langle w_{z}\right\rangle$ linearly evolves according to a diffusion equation:

$$
\tilde{\partial}_{t}\left\langle w_{z}\right\rangle \sim-A \tilde{\partial}^{2}\left\langle w_{z}\right\rangle,
$$

where $A$ is a positive coefficient representing the linear eddy-viscosity tensor of (3.6), restricted to low Deborah numbers (as explained in the end of $\S 3$. It vanishes on the stability curve, being positive above it and negative below it.

In this equation, all modes are linearly unstable. It needs to be modified to keep track of the multiscale hypothesis, which requires small-scale modes to be stable. This is done introducing a bi-Laplacian term into (4.2) to stabilize the small scales (the fourth-order derivative ensures that this term will be dominant on the small-scale perturbations only):

$$
\tilde{\partial}_{t}\left\langle w_{z}\right\rangle \sim-A \tilde{\partial}^{2}\left\langle w_{z}\right\rangle-C \tilde{\partial}^{4}\left\langle w_{z}\right\rangle .
$$

In general, close to the linear instability threshold, where the coefficient $A$ changes sign, we do not expect $C$ to vanish. To comply with the stability requirements, we will request it to have a finite, positive value in the region of interest.

We expect to find the presence of a nonlinear term, eventually stabilizing this growth. This part cannot be played by the advective nonlinearity because of the one-dimensional character of the equation. The next-order nonlinearity is cubic and must contain at least two space derivatives: one before the whole term, to ensure momentum conservation, and an additional one to respect space-inversion symmetries. This yields a nonlinear term: $B \tilde{\partial}_{x}\left(\left\langle w_{z}^{(1)}\right\rangle^{2} \tilde{\partial}_{x}\left\langle w_{z}^{(1)}\right\rangle\right)$, where $B$ is some constant related to the (nonlinear) eddyviscosity.

We can now introduce, as in $\S[$, the "slow" variables $\tilde{\boldsymbol{x}}=\epsilon \boldsymbol{x}$ and $\tilde{t}$ (notice that we still do not know the scaling between $t$ and $\tilde{t}$ ). The space derivatives must again be expanded as $\partial_{i} \rightarrow \partial_{i}+\epsilon \tilde{\partial}_{i}$. We now have to look for a prescription on how to expand the different fields in terms of $\epsilon$.

In the vicinity of the marginal eddy-viscosity curve $A \approx 0$, and a Taylor expansion gives $\left.A \sim \frac{\partial A}{\partial \nu}\right|_{\nu_{c}}\left(\nu-\nu_{c}\right)$, where $\nu_{c}$ indicates the critical viscosity. Balances between the term $A \tilde{\partial}^{2}\left\langle w_{z}\right\rangle$ and both the cubic nonlinearity and $C \tilde{\partial}^{4}\left\langle w_{z}\right\rangle$ yield:

$$
\left.B \epsilon^{2} \epsilon_{w}^{3} \sim \frac{\partial A}{\partial \nu}\right|_{\nu_{c}}\left(\nu-\nu_{c}\right) \epsilon^{2} \epsilon_{w},\left.\quad \frac{\partial A}{\partial \nu}\right|_{\nu_{c}}\left(\nu-\nu_{c}\right) \epsilon^{2} \epsilon_{w} \sim C \epsilon^{4} \epsilon_{w} .
$$

Here, $\epsilon_{w}$ is the scaling of the amplitude of the velocity perturbation $\boldsymbol{w}$.

Equations (4.3) completely define the scaling for the velocity perturbation and the 
distance from the critical viscosity:

$$
\epsilon=\epsilon_{w}, \quad \frac{\nu-\nu_{c}}{\nu} \sim \epsilon^{2} \Rightarrow \nu=\nu_{c}\left(1-\epsilon^{2}\right) .
$$

It follows from (4.4) that the Reynolds number $R e=R e_{c}\left(1+\epsilon^{2}\right)$. The comparison of any of the previous terms with the (slow) time derivative of $\boldsymbol{w}$ gives the scaling $\tilde{t}=\epsilon^{4} t$. As for the scaling of the polymer conformation tensor, balancing $\zeta / \tau$ and $\left(\partial w_{z}\right) \cdot \sigma$, we obtain that the scaling of $\boldsymbol{\zeta}$ coincides with $\epsilon_{w}$. The same equality holds for the pressure field.

Summarizing, the fields are expanded as:

$$
\begin{aligned}
& \boldsymbol{w}=\epsilon \boldsymbol{w}^{(1)}(z, \tilde{x}, \tilde{t})+\epsilon^{2} \boldsymbol{w}^{(2)}(z, \tilde{x}, \tilde{t})+\ldots, \\
& q=\epsilon q^{(1)}(z, \tilde{x}, \tilde{t})+\epsilon^{2} q^{(2)}(z, \tilde{x}, \tilde{t})+\ldots, \\
& \boldsymbol{\zeta}=\epsilon \boldsymbol{\zeta}^{(1)}(z, \tilde{x}, \tilde{t})+\epsilon^{2} \boldsymbol{\zeta}^{(2)}(z, \tilde{x}, \tilde{t})+\ldots
\end{aligned}
$$

The next step to obtain a coarse-grained equation for the large-scale dynamics is to plug (4.5) into (3.1)- Exploiting the chain rule, the definitions of $\tilde{x}$ and $\tilde{t}$ and averaging along $z$, we end up with a set of equations involving solely the large-scale fields. The equation for the large-scale transverse perturbation $\left\langle w_{z}^{(1)}\right\rangle(\tilde{x}, \tilde{t})$ is obtained from the solvability condition at order $\epsilon^{5}$. For details on the Newtonian case and solvability conditions, see Gama et al. (1994).

We can summarize the whole procedure in the following schematic way:

(a) Solve the continuity equation. The explicit expression of $w_{z}^{(n)}$ is thus obtained in terms of known functions of $z$.

(b) Solve the equation for $\zeta_{z z}^{(n)}$; this can always be done algebraically as $\zeta_{z z}^{(n)}$ is slaved to the $w_{z}^{(n)}$ field.

(c) Solve the evolution equation for $w_{z}^{(n)}$. This field is obtained from $(a)$; we are then able to obtain the expression for the pressure field perturbation $q$.

(d) Solve the system for $\zeta_{x z}^{(n)}$ and $w_{x}^{(n)}$ by direct integration.

(e) Algebraically obtain the explicit expression for $\zeta_{x x}^{(n)}$.

(f) Impose solvability condition at order $n+1$ on the continuity and the velocity field equations. Such condition is automatically fulfilled by the polymer conformation tensor, as it is slaved to the velocity field at the previous order.

The final equation has the form of a "standard" Cahn-Hilliard equation:

$$
\tilde{\partial}_{t}\left\langle w_{z}^{(1)}\right\rangle=\tilde{\partial}_{x}\left[\left(-A+B\left\langle w_{z}^{(1)}\right\rangle^{2}\right) \tilde{\partial}_{x}\left\langle w_{z}^{(1)}\right\rangle\right]-C \tilde{\partial}_{x}^{4}\left\langle w_{z}^{(1)}\right\rangle
$$

"Standard" is meant to stress that the structure of (4.6) (including the cubic nonlinearity) emerges in a variety of hydrodynamic situations (see Nepomnvashchvi 1976; Sivashinskv 1985; Pedloskv 1987; Manfroi \& Young 1999). The parameters $A, B, C$ are known functions of the parameters $D e$ and $\beta$, as shown in figure 2 It is worth noting that $A$ is non-negative as the system is supposed to be slightly above the threshold of instability and we have explicitly incorporated a negative sign in (4.6).

The saturation of the instability requires two conditions. First, $C$ must be positive to ensure that the instability be saturated at sufficiently high wave-numbers (still much smaller than those of the basic flow, of order unity). Second, $B$ ought to be positive to ensure that, as $\left\langle w_{z}^{(1)}\right\rangle$ becomes $O(\sqrt{A / B})$, the nonlinear eddy-viscosity $-A+B\left\langle w_{z}^{(1)}\right\rangle^{2}$ change sign and the growth be again saturated. Both these conditions are satisfied up to a critical value of the Deborah number, $D e^{*}$ (see figure 2). 


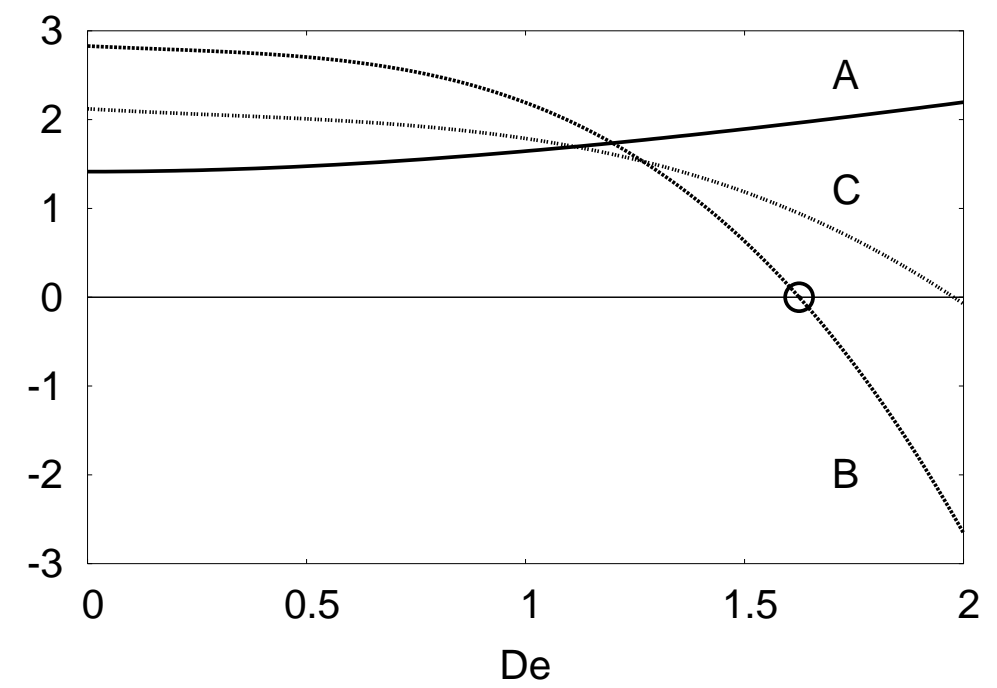

Figure 2. The parameters $A, B$ and $C$ appearing in the coarse-grained Cahn-Hilliard equation (4.6) as a function of the Deborah number (for $\beta=0.769$ ).

Up to the critical Deborah number $D e^{*}$, the equation (4.6) for the large-scale perturbations has the same structure as in the Newtonian case and the only difference is in the numerical value of the parameters $A, B, C$. As we shall see in the next sections, this property ceases to be true above $D e^{*}$.

To conclude, we stress the fact that all the fields up to order four are expressed in terms of explicit functions of the fast variables and of the large-scale field $\left\langle w_{z}^{(1)}\right\rangle$, obeying the Cahn-Hilliard equation (4.6).

\section{Generalized Cahn-Hilliard dynamics}

We have observed in the previous section that, along the marginal linear stability curve, there exists a critical value of the Deborah number, $D e^{*}$, where the cubic nonlinear term becomes negative. Furthermore, the change of sign is taking place in the region where the small-scale operator is stable. The problem is thus well-posed and lends to multiscale methods.

For $D e>D e^{*}$, the field keeps growing at sufficiently large scales, until it reaches amplitudes where the next-order nonlinearity becomes important. Its structure is dictated by the conservation of momentum and the symmetries of the basic flow: $\tilde{\partial}_{x}\left(\left\langle w_{z}\right\rangle^{4} \tilde{\partial}_{x}\left\langle w_{z}\right\rangle\right)$, with a regular coefficient $D$ in the neighborhood of the critical point $P^{*}$, where both the eddy-viscosity and the coefficient of the third-order nonlinearity change sign.

Four regions can be identified around $P^{*}$ (see figure 3). The eddy-viscosity $A=0$ curve has been obtained by means of the linear stability analysis $(\S 3)$. The linear approximation of the curve $B=0$ in the vicinity of $P^{*}$ is obtained from the analytic expression of $B$ on the marginal curve and the marginal curve itself.

Zone I is linearly unstable $(A>0)$, has a third-order destabilizing term $(B<0)$ and we can guess that a fifth-order term will enter into play to stabilize the growth. Zone II is particularly interesting as it is linearly stable $(A<0)$, but has a third-order destabilizing contribution $(B<0)$. Perturbing with a field of sufficiently strong amplitude, the system 
10 A. Bistagnino, G. Boffetta, A. Celani, A. Mazzino, A. Puliafito, M. Vergassola

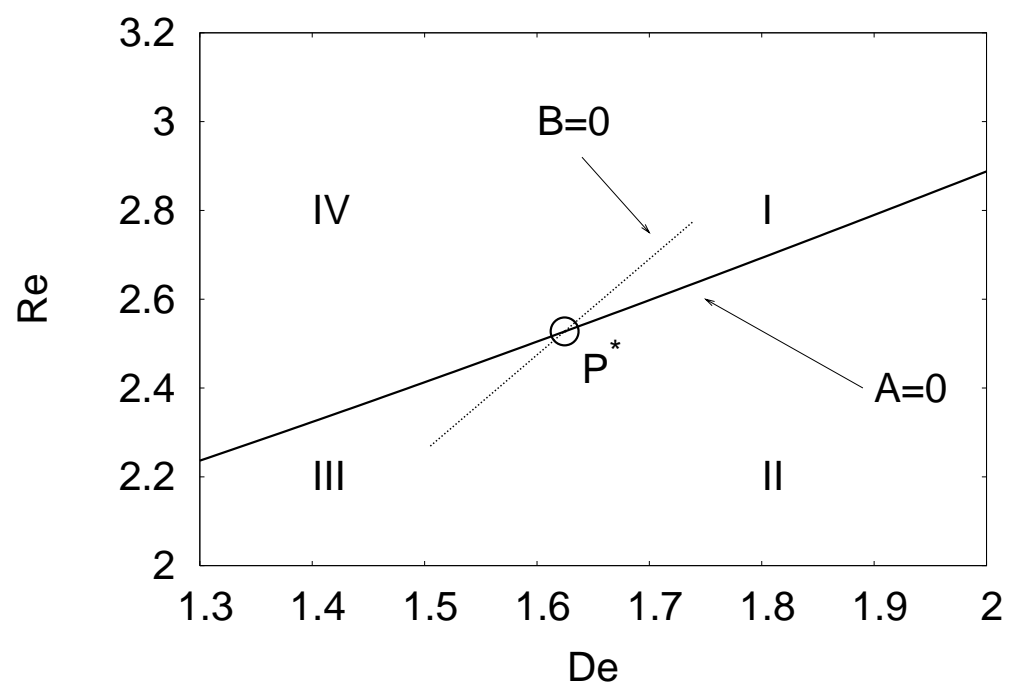

FiguRE 3. The phase-space around the critical point $P^{*}$ where both the eddy-viscosity and the coefficient of the third-order nonlinearity change sign. The region is divided in four regions schematically sketched here by the two critical curves $A=0$ and $B=0$. The former is found from the linear stability analysis in section 3 . The latter is found locally, around the $A=0$ curve, by solving (5.5), and is linearly extrapolated for graphical purposes as a dashed line. For $\beta=0.769$, the curve $B=0$ is inclined at approximately $60^{\circ}$ with respect to the $D e$ axis.

jumps to the asymptotic steady state where the two nonlinear terms (third and fifthorder) balance each other. Zone III is completely stable $(A<0, B>0)$. In the last region, IV, as $D e$ approaches the critical value, the coefficient $B$ goes to zero and cannot saturate the exponential growth from the linear instability. The fifth-order nonlinearity, which is negligible far from the critical point, must enter again into play.

\subsection{Zone $I$}

When both the Reynolds and the Deborah numbers exceed their critical values, previous considerations suggest the following structure for the coarse-grained equation:

$$
\tilde{\partial}_{t} w=-A \tilde{\partial}_{x}^{2} w-|B| \tilde{\partial}_{x}\left(w^{2} \tilde{\partial}_{x} w\right)-C \tilde{\partial}_{x}^{4} w+D \tilde{\partial}_{x}\left(w^{4} \tilde{\partial}_{x} w\right) .
$$

Confining the analysis to the surroundings of the critical point $P^{*}$, we may represent the position in phase space as:

$$
\begin{array}{r}
\nu=\nu^{*}\left(1-K_{1} \epsilon_{\nu}-K_{2} \epsilon_{\nu}^{2}\right), \\
D e=D e^{*}\left(1+\epsilon_{D e}\right) .
\end{array}
$$

Adequately choosing the $K_{1}$ and $K_{2}$ parameters, any point around $P^{*}$ can be reached as $\epsilon$ varies. The reason why we need to incorporate in (5.2) the additional contribution of order $\epsilon^{2}$ will be clear shortly.

In the neighborhood of $P^{*}$, the coefficients $A$ and $B$ are expanded as:

$$
\begin{aligned}
& A=\frac{\partial A}{\partial D e}\left(D e-D e^{*}\right)+\frac{\partial A}{\partial \nu}\left(\nu-\nu^{*}\right), \\
& B=\frac{\partial B}{\partial D e}\left(D e-D e^{*}\right)+\frac{\partial B}{\partial \nu}\left(\nu-\nu^{*}\right),
\end{aligned}
$$

where all derivatives are computed at $P^{*}$. 
The scaling in $\epsilon$ of the velocity field amplitude, $\epsilon_{w}$, and the parameters $\epsilon_{\nu}, \epsilon_{D e}$ is found by requiring that all terms in (5.1) be of the same order in the scale-separation small parameter $\epsilon$.

The comparison between the last two terms in (5.1) fixes the relation between $\epsilon$ and $\epsilon_{w}$ :

$$
D \epsilon^{2} \epsilon_{w}^{5} \sim C \epsilon^{4} \epsilon_{w} \Rightarrow \epsilon_{w}=\epsilon^{1 / 2} .
$$

The parameters $\epsilon_{\nu}$ and $\epsilon_{D e}$ are found by comparing the terms associated to $A, B$ and $D$ in (5.1). Using (5.2)-(5.6), we obtain:

$$
\begin{aligned}
D \epsilon^{2} \epsilon^{5 / 2} & \sim\left[\frac{\partial A}{\partial D e}\left(\epsilon_{D e} D e^{*}\right)-\frac{\partial A}{\partial \nu}\left(K_{1} \epsilon_{\nu} \nu^{*}\right)\right] \epsilon^{2} \epsilon^{1 / 2} \\
D \epsilon^{2} \epsilon^{5 / 2} & \sim\left[\frac{\partial B}{\partial D e}\left(\epsilon_{D e} D e^{*}\right)-\frac{\partial B}{\partial \nu}\left(K_{1} \epsilon_{\nu} \nu^{*}\right)\right] \epsilon^{2} \epsilon^{3 / 2} .
\end{aligned}
$$

Choosing $\epsilon_{\nu}=\epsilon_{D e}=\epsilon$ and setting $K_{1}$ to ensure $\left[\frac{\partial A}{\partial D e} D e^{*}-\frac{\partial A}{\partial \nu} K_{1} \nu^{*}\right]=0$, both equations (5.7) and (5.8) are satisfied. Equation (5.7) is balanced by the second-order term of the $\nu$ expansion (5.2), dependent on $K_{2}$. The scalings of time, pressure and polymer conformation tensor perturbation, $\epsilon^{4}, \epsilon^{1 / 2}$ and $\epsilon^{1 / 2}$, respectively, are derived as in $\S$ 团

Once the scalings have been determined we can proceed as in $\S$ to obtain the largescale equation for $\left\langle w_{z}^{(1 / 2)}\right\rangle(\tilde{t}, \tilde{x})$. The evolution equation emerges now from the solvability condition at the order $\epsilon^{9 / 2}$ :

$$
\tilde{\partial}_{t}\left\langle w_{z}^{(1 / 2)}\right\rangle=\tilde{\partial}_{x}\left[\left(-A+B\left\langle w_{z}^{(1 / 2)}\right\rangle^{2}+D\left\langle w_{z}^{(1 / 2)}\right\rangle^{4}\right) \tilde{\partial}_{x}\left\langle w_{z}^{(1 / 2)}\right\rangle\right]-C \tilde{\partial}_{x}^{4}\left\langle w_{z}^{(1 / 2)}\right\rangle,
$$

where the coefficients are explicit functions of $\beta$. For $\beta=0.769$, they read:

$$
\left\{\begin{array}{l}
A=0.5106+1.965 K_{2}, \quad B=-8.979 \\
C=0.9439, \quad D=23.11, \quad K_{1}=0.594
\end{array}\right\}
$$

Although (5.9) belongs to the class of the Cahn-Hilliard equations (1.1), the emergence of the new, sixth-order nonlinearity will be responsible for new dynamical aspects, not present for $D e<D e^{*}$, which will be discussed in detail in $\S[$ ]

\subsection{Zone $I I$}

For Deborah numbers above the critical value, perturbations are nonlinearly unstable: $B<0$. This is true regardless of the sign of the linear term and strong enough perturbations may then grow even if the system is linearly stable.

Let us then consider systems with $\nu>\nu^{*}$ and $D e>D e^{*}$. No major difference with respect to case I is expected. At zero-th order, the coefficients $A$ and $B$ vanish and equations (5.4)-(5.5) hold. Again, we define the position in phase-space via the two parameters $\epsilon_{\nu}$ and $\epsilon_{D e}$. As the viscosity is now larger than the critical value, a positive sign appears in the expansion of the viscosity:

$$
\nu=\nu^{*}\left(1+K_{1} \epsilon_{\nu}-K_{2} \epsilon_{\nu}^{2}\right),
$$

while (5.3) holds. The parameter $K_{2}$, as we shall point out later, can take any value compatible with the condition $A>0$.

The same calculations discussed in the previous subsection can be carried out to derive the coarse-grained equation for the transverse velocity. As one might expect, its form is exactly the same as (5.9), a generalized Cahn-Hilliard equation. The only difference is 
12 A. Bistagnino, G. Boffetta, A. Celani, A. Mazzino, A. Puliafito, M. Vergassola

in the value of the parameters. For $\beta=0.769$, they read:

$$
\left\{\begin{array}{l}
A=-0.2202+1.965 K_{2}, \quad B=-35.62 \\
C=0.9439, \quad D=23.11, \quad K_{1}=0.5974
\end{array}\right\}
$$

Only $A$ and $B$ have changed with respect to (5.10), as expected since they are the only parameters which depend on $\epsilon$ (and thus on $R e$ and $D e$ ) in physical coordinates. Notice that there is an upper bound on the values we can choose for $K_{2}$, reflecting the linear stability requirement.

\subsection{Zone $I V$}

What happens when the Deborah number is barely smaller than the critical value $D e^{*}$ ? Sufficiently close to it, the third-order instability can be made subdominant with respect to the fifth-order and our aim here is to work out the scaling coefficients corresponding to such situation.

For this purpose, let us assume that the cubic nonlinearity is negligible. At leading order, the terms associated to $A, C$ and $D$ must be of the same order. This means:

$$
\begin{aligned}
\epsilon^{4} \epsilon_{w} & \sim \epsilon^{2} \epsilon_{w}\left[\frac{\partial A}{\partial D e}\left(D e-D e^{*}\right)+\frac{\partial A}{\partial \nu}\left(\nu-\nu^{*}\right)\right], \\
\epsilon^{2} \epsilon_{w}^{5} & \sim \epsilon^{2} \epsilon_{w}\left[\frac{\partial A}{\partial D e}\left(D e-D e^{*}\right)+\frac{\partial A}{\partial \nu}\left(\nu-\nu^{*}\right)\right],
\end{aligned}
$$

and implies:

$$
\nu=\nu^{*}\left(1-K_{2} \epsilon^{2}\right), \quad D e=D e^{*}\left(1-\epsilon^{2}\right) .
$$

Additionally, the velocity field scales as $\epsilon^{1 / 2}$, as the pressure and polymer fields do. The time derivative scales as $\epsilon^{4}$.

To be consistent, we are left to check that the third-order nonlinearity is negligible. Using the previous scalings and the ensuing fact that $B \sim O\left(\epsilon^{2}\right)$, we have to verify that:

$$
O\left(B \partial^{2} w^{3}\right) \ll O\left(D \partial^{2} w^{5}\right) \Rightarrow O\left(\epsilon^{11 / 2}\right) \ll O\left(\epsilon^{9 / 2}\right),
$$

which holds true. It is now possible to apply the strategy discussed in $\S \square$ to derive the large-scale equation and obtain (at order $\epsilon^{5}$ ):

$$
\tilde{\partial}_{t}\left\langle w_{z}^{(1 / 2)}\right\rangle=\tilde{\partial}_{x}\left[\left(-A+D\left\langle w_{z}^{(1 / 2)}\right\rangle^{4}\right) \tilde{\partial}_{x}\left\langle w_{z}^{(1 / 2)}\right\rangle\right]-C \tilde{\partial}_{x}^{4}\left\langle w_{z}^{(1 / 2)}\right\rangle,
$$

where $C$ and $D$ have the same value as before and $A=1.1740+1.965 K_{2}$.

\section{Variational formulation}

It is well-known that the "standard" Cahn-Hilliard equation admits a variational formulation in terms of a Ginzburg-Landau potential (Cahn \& Hilliard 1958). Equation (4.6), after appropriate rescalings, $w \rightarrow(A / B)^{1 / 2} w, t \rightarrow A^{-1} t, \lambda=C / A$, is recast in the form (1.1) with the Lyapunov functional:

$$
F[w]=\int\left[\frac{\lambda}{2}\left(\partial_{x} w\right)^{2}+I(w)\right] \mathrm{d} x, \quad I(w)=-\frac{w^{2}}{2}+\frac{w^{4}}{12} .
$$

Note that mean fields only are considered, that is $w$ must be read as the rescaled leading contribution $\left\langle w_{z}^{(1)}\right\rangle(\tilde{x}, \tilde{t})$.

The existence of a Lyapunov functional implies the existence of an asymptotic state 
for $w$, if boundary conditions are periodic and stationary. Such state corresponds to a minimum of the Lyapunov functional and it is calculated by the following equations:

$$
I^{\prime}(w)=\lambda \partial_{x}^{2} w \leftrightarrow \partial_{x} I=\frac{\lambda}{2} \partial_{x}\left(\partial_{x} w\right)^{2} .
$$

When $w$ is a maximum (or a minumum), $I\left(w_{\max }\right)$ is constant and $w_{\max }$ is obtained solving $I^{\prime}=0$ as $w_{\max }= \pm \sqrt{3}$. Considering this boundary condition, equation (6.2) can be easily solved. Its solutions are the well-known kink and anti-kink structures, namely:

$$
w= \pm \sqrt{3} \tanh \left[\sqrt{\frac{1}{2 \lambda}} x\right] .
$$

The issue now is whether or not a Lyapunov extremal formulation exists in the generalized Cahn-Hilliard case (5.1) as well, and how it relates to the standard one. In particular, a Painlevé test (Ablowitz \& Clarkson 1991) can be performed on the equation to check its integrability. The calculation consists in checking that all movable singularities (whose location depends on initial and/or boundary conditions) are poles (see for details Ablowitz \& Clarkson 1991). The test is based on a well-known connection between the integrability property of a nonlinear differential equation and its analytic structure for complex values of the independent variable (Kowalesvki 1889, 1890; Painlevé 1897). The explicit calculation is performed in Appendix B The generalized Cahn-Hilliard equation indeed enjoys the Painlevé property and is thus integrable.

Let us then write the equation (5.1) after the rescalings $w \rightarrow(A / B)^{1 / 2} w, t \rightarrow$ $A^{-1} t, \lambda=C / A, \gamma=A D / B^{2}$ :

$$
\partial_{t} w=-\partial_{x}^{2} w-\frac{1}{3} \partial_{x}^{2} w^{3}-\lambda \partial_{x}^{4} w+\frac{\gamma}{5} \partial_{x}^{2} w^{5} .
$$

Integrability of this equation is related to the existence of the following Lyapunov functional, similar to that of the standard case, yet with a sixth-order potential:

$$
F[w]=\int\left[\frac{\lambda}{2}\left(\partial_{x} w\right)^{2}+I(w)\right] \mathrm{d} x, \quad I(w)=-\frac{w^{2}}{2}-\frac{w^{4}}{12}+\frac{\gamma}{30} w^{6} .
$$

The calculation of the function corresponding to its minimum is performed using again (6.2).

All solutions tend to final steady states which minimize $F$. The approach to the solution is however nontrivial and the structure is made of plateaux having velocity $\pm W_{0}$ $\left(I^{\prime}\left(W_{0}\right)=0\right)$, separated by positive and negative kinks (see figure 4). The amplitude of the velocity $w$ in the plateaux is:

$$
W_{0}^{2}=\frac{5+\sqrt{25+180 \gamma}}{6 \gamma}
$$

Note that, at small $\gamma$ 's, the asymptotic velocity $W_{0}$ diverges as $1 / \sqrt{\gamma}$. This is quite intuitive: the field amplitude equilibrating the third and the fifth-order nonlinearities increases as the coefficient of the fifth-order nonlinearity reduces.

The explicit expression of the profiles for kinks and anti-kinks is obtained from the integration of equations (6.2), (6.5). For the sake of example, when $\lambda=1 / 2$ and $\gamma=10 / 9$ the profiles read:

$$
w= \pm \sqrt{15} \frac{e^{2 \sqrt{3} x}-1}{\sqrt{5 e^{4 \sqrt{3} x}+26 e^{2 \sqrt{3} x}+5}} .
$$


14 A. Bistagnino, G. Boffetta, A. Celani, A. Mazzino, A. Puliafito, M. Vergassola

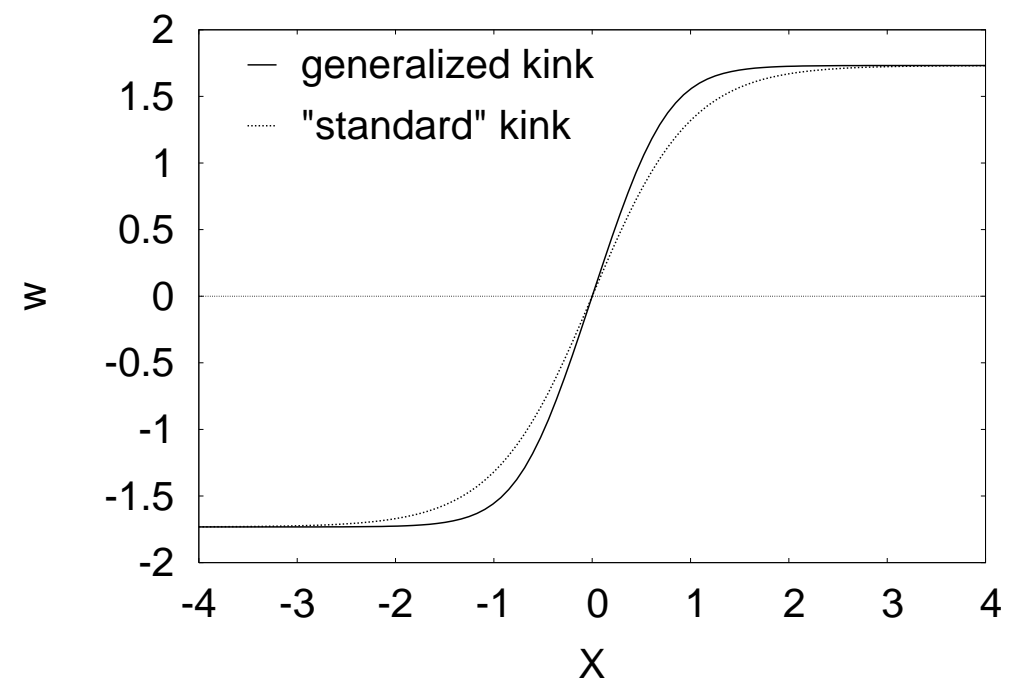

Figure 4. The "generalized" (solid) and "standard" (dotted) kinks for $\lambda=1 / 2$ and $\gamma=10 / 9$. The former has a manifestly shorter range. It is shown in the body of the text that this entails longer time-scales for their annihilation with the corresponding anti-kinks.

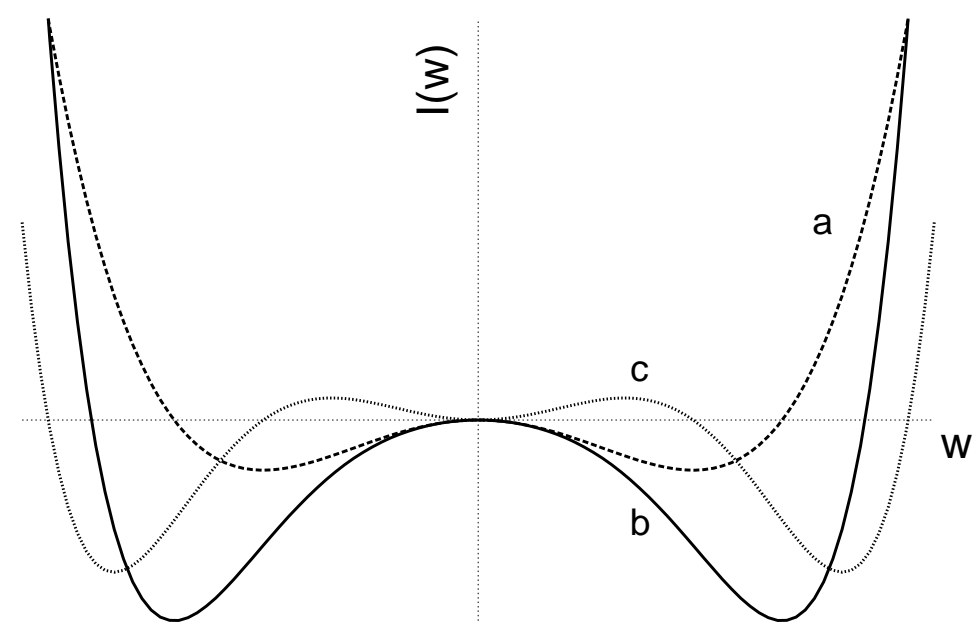

Figure 5. The potentials associated to the different evolution equation. Curve $a$ is related to the standard Cahn-Hilliard equation (fourth-order potential); curve $b$ represents the generalized Cahn-Hilliard equation (sixth-order potential). Curve $c$ is the characteristic triple-well potential of the purely nonlinearly unstable case. The plots are in arbitrary units, to ease the comparison between the curves.

The generalized kink-antikink structures, e.g. those given by equation (6.7), will be dubbed "generalized" kinks and anti-kinks. 
6.1. Dynamics of generalized kink/antikink annihilation and approach to equilibrium

Detailed calculations are performed following Legras \& Villone (2003), who in turn based theirs on Kawasaki \& Ohta (1982). They are lengthy, yet quite simple in their basic idea, sketched hereafter.

During metastable transitions, the kinks do not satisfy (6.2) exactly, due to the presence of other kinks and/or anti-kinks. The deviation of the amplitude in the plateau is proportional to $e^{-s \Lambda}$, where $\Lambda=4|x|$ and $x$ denotes the distance to the point where $w=0$. Here, $s$ is the inverse of the typical length scale of this deviation (for details, see Appendix A of Legras \& Villone 2003). The quantity $s$ turns out to be crucial as neighboring kinks and anti-kinks attract proportionally to $e^{-s \Delta x}$, where $\Delta x$ is the distance between neighbouring kinks and anti-kinks (for details, see Appendix B of Legras \& Villone 2003).

The behavior of the kink size $s$ is grasped as follows. Consider a metastable state of the Cahn-Hilliard equation. The potential felt by a kink $w(x)$ close to the plateau $w=W_{0}$ is estimated by the Taylor expansion:

$$
I\left(w-W_{0}\right) \simeq I\left(W_{0}\right)+I^{\prime}\left(W_{0}\right)\left(w-W_{0}\right)+I^{\prime \prime}\left(W_{0}\right) \frac{\left(w-W_{0}\right)^{2}}{2},
$$

where we know that $I^{\prime}\left(W_{0}\right)=0$. Note also that the dynamics of $w$ does not change if we add an arbitrary constant to the potential $I$, so that we can set $I\left(W_{0}\right) \equiv 0$.

Let us now calculate the shape of the profile between $w$ and $W_{0}$. For a metastable state, $\partial_{t}\left(w-W_{0}\right)=0$, that implies:

$$
\frac{\lambda}{2}\left(\partial_{x}\left(w-W_{0}\right)\right)^{2}+\left[I^{\prime \prime}\left(W_{0}\right) \frac{\left(w-W_{0}\right)^{2}}{2}\right]=0 .
$$

Interpreting $\partial_{x}$ as the inverse of the typical length scale $s$ for $\left(w-W_{0}\right)$, one easily obtains $s=\sqrt{\lambda / I^{\prime \prime}\left(W_{0}\right)}$. The second-order derivative can be explicitly calculated using (6.6):

$$
I^{\prime \prime}\left(W_{0}\right)=4+\frac{2}{3} W_{0}^{2} .
$$

Qualitative properties of $s$ are easy to grasp. At large $\gamma$ 's, the size of the kinks tends to a constant, independent of $\gamma$. At small $\gamma$ 's, the kinks get steeper and steeper, their size scaling as $\gamma^{1 / 2}$. This implies that the convergence to equilibrium will be slower and slower as $\gamma$ is reduced (recall that the kinks attract proportionally to $e^{-s \Delta x}$ ).

For the same band of unstable modes, i.e. keeping $\lambda$ fixed, it holds that the convergence to equilibrium is slower for the generalized than for the standard Cahn-Hilliard equation. Indeed, for the Cahn-Hilliard potential $I_{C H}=-w^{2} / 2+w^{4} / 12$, the second-order derivative $I_{C H}^{\prime \prime}\left(W_{0}\right)=2$. As for (6.10), we can use the identity $1+W_{0}^{2} / 3=\gamma W_{0}^{4} / 5$, following from the very definition $I^{\prime}\left(W_{0}\right)=0$, to obtain $I^{\prime \prime}\left(W_{0}\right)>2$. This implies that the interactions for the generalized kink-antikink structures have a shorter range and their dynamics of annihilation is thus slower.

A special remark applies to the linearly stable case (zone II). In this case, the equation is associated to an uncommon triple-well potential. The typical nonlinear kink-antikink dynamics appears only if the initial perturbation will be energetic enough to let the system "jump" out of the central well and fall into one of the side wells.

\section{Numerical results}

The analytical results presented in this work have been obtained by multiscale techniques. Their basic assumption is the strong scale separation between the basic flow and 


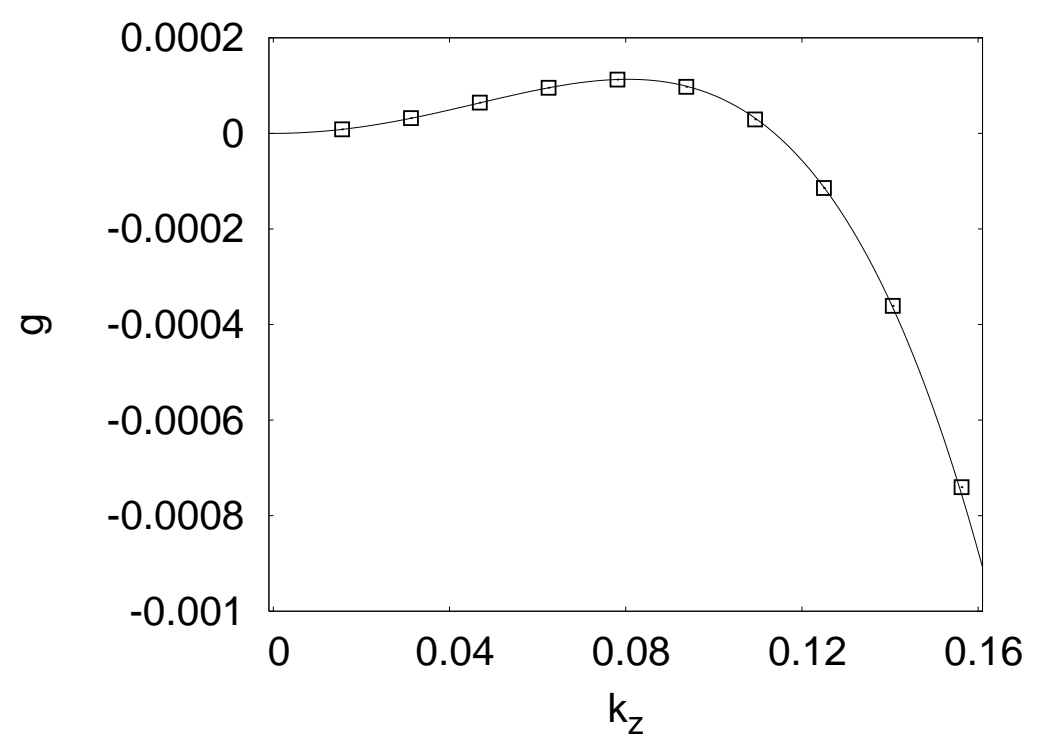

FiguRE 6. Growth rates $g$ of the transverse Fourier modes $k$ for a simulation with $D e=1.4$ and $\beta=0.769$. The simulations are performed in a rectangular domain with aspect ratio $1 / 64$. The distance to the critical point $R e / R e_{c}-1$ is 0.28 . The solid line represents the linear prediction (7.1). The circles representing the numerically computed growth rates have been obtained with a DNS simulation by a linear fit of the logarithm of the energy for each mode versus time, in the early stages of their exponential growth.

the most unstable perturbations. In this section, we shall present the numerical simulations performed to check the validity of this assumption.

The linear analysis results have already been checked in Boffetta et al. (2005a) by reducing the original linear partial differential equation to a generalized eigenvalue problem and computing its eigenvalues/eigenvectors. The scale separation hypothesis is found to be well verified up to Deborah numbers of order unity ( $D e \approx 2.3$ for the value $\beta=0.769$ used in this study). For larger $D e$, scale separation does not hold and multiscale methods are not applicable.

To check the nonlinear results derived here, we have numerically simulated the complete Oldroyd-B model equations (2.2)-(2.4) via a pseudospectral algorithm (see Canuto et al. 1988, for details on the numerical method). In the following, we will refer to these as to Direct Numerical Simulations (DNS), while numerical integration of the one-dimensional Cahn-Hilliard equation (4.6) will be referred to as $\mathrm{CH}$ simulations.

To enforce a transverse perturbation, we integrated the equations on a rectangular slab with $L=L_{x}=2 \pi$ and $L_{z}=64 L_{x}$. The aspect ratio $r$ is then fixed at $1 / 64$. The simplest check of our results concerns the growth rates of the instability which, in the linear regime, can be obtained by the Cahn-Hilliard equation. Neglecting the nonlinear term, the dispersion relation for the transverse Fourier modes $k$ reads:

$$
g=A\left(\frac{R e}{R e_{c}}-1\right) k^{2}-C k^{4}
$$

In figure 6] we report the growth rates of the first modes for a (white-noise in space) small initial perturbation. We are then able to observe also negative $g$ (stable modes). The comparison with the linear prediction is excellent, even for modes whose scale separation is not very small. 


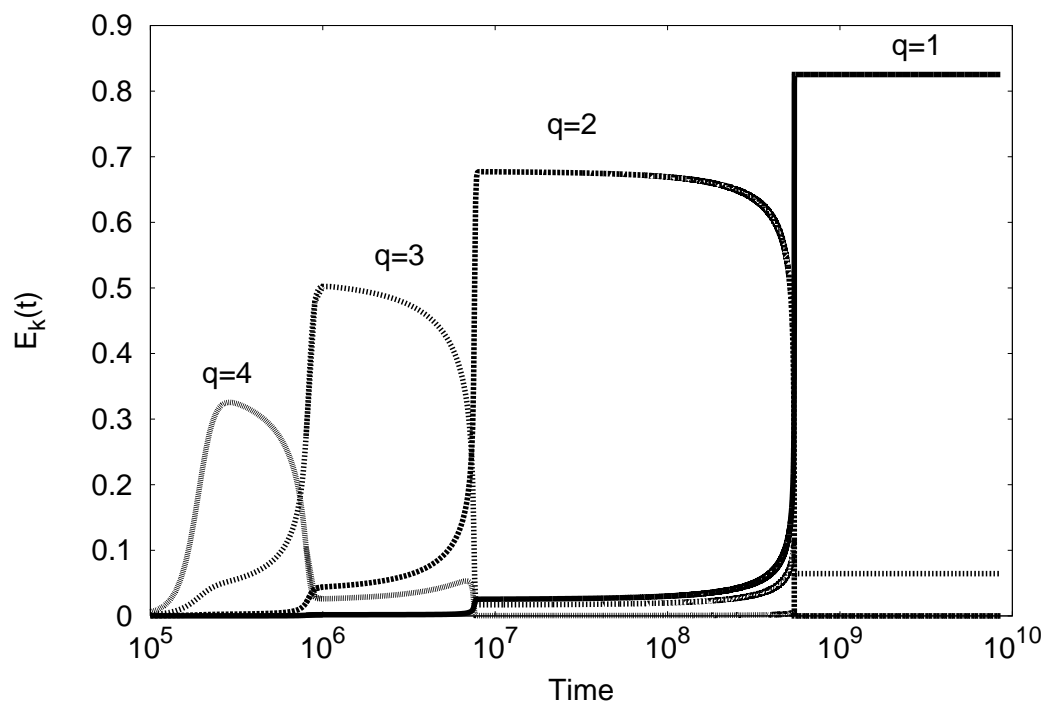

FiguRE 7. The energy associated to the lowest wavenumber modes resulting from a $\mathrm{CH}$ simulation. The values of the coefficients have been arbitrarily chosen for conveniency of display. The quasi-stationary states can be clearly seen up to the asymptotic one corresponding to the largest periodicity. In this simulation, $D e=1.4$ and $R e / R e_{c}-1=0.28$.

Let us now consider the nonlinear stage of the perturbation growth. It is well known that the time evolution of the Cahn-Hilliard equation shows a succession of long-lasting metastable states characterized by a well defined periodicity. For sufficiently small initial perturbations, the wave-number $k$ associated to the maximum growth-rate $g$ will be the first to reach the balance between the destabilizing linear term $A \tilde{\partial}^{2}\left\langle w_{z}\right\rangle$ and the stabilizing non-linear one $B \tilde{\partial}^{2}\left\langle w_{z}\right\rangle^{3}$. When such equilibrium is reached, the energy associated to that mode is constant and the system is quasi-stable. In the meanwhile the other modes $k_{\max }-1, k_{\max }-2, \ldots$ keep growing. When the mode $k_{\max }-1$ balances the two terms, the energy associated to the mode $k_{\max }$ drops. This new state is again quasi-stationary and has a well-defined periodicity $k_{\max }-1$.

The process continues until a state with the box periodicity is reached (see figure 7); such a state is stationary and corresponds to the asymptotic behaviour in $\S[$. The kink structures described there, are characteristic of all of these stages. Indeed, any transition between two quasi-stationary states can be seen as a kink-antikink annihilation, yielding a decrease in periodicity, as in figure 8 (She 1987).

To check the results obtained in $\S \square$ we have performed a DNS simulation for a particularly long lapse of time. The excellent agreement between the DNS and the prediction of the Cahn-Hilliard equation is shown in figure 9

The same comparison can be realized in the neighborhood of the critical point $P^{*}$. This kind of simulation is much harder than for the standard Cahn-Hilliard, because it involves a very precise knowledge of the position of the critical point, and there is no easy way to obtain this from the simulations. Moreover, any system we simulate will be at a finite distance from the critical point. The parameter that will mostly feel this difference will be $D$, as we have chosen it to be approximately constant around $P^{*}$. We have been able to overcome this weakness via a limited tweaking of the $D$ parameter in the $\mathrm{CH}$ simulation. As shown in figure [10] an excellent agreement between the curves is again achieved. 
18 A. Bistagnino, G. Boffetta, A. Celani, A. Mazzino, A. Puliafito, M. Vergassola

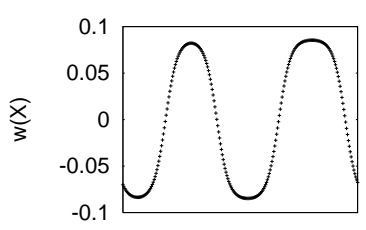

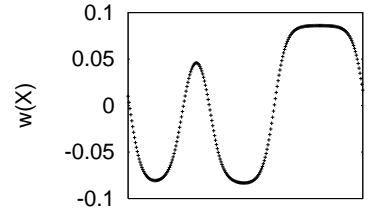

$\mathrm{X}$

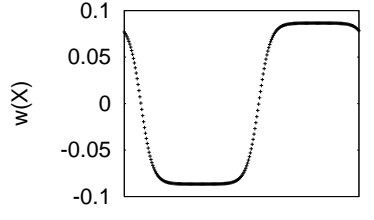

$\mathrm{X}$

FIGURE 8. Instantaneous transverse velocity field at different times. The simulation is the same as in figure 7 The transition between two metastable states can be regarded as a kink-antikink annihilation. In this figure a transition from a $k=2$ to a $k=1$ state is represented, the $x$-axis being the physical $x$ direction of the integration box and the $y$-axis being the amplitude of the $w$ perturbation. The time figure set over the graphs refers to the evolution shown in figure $\mathbf{7}$

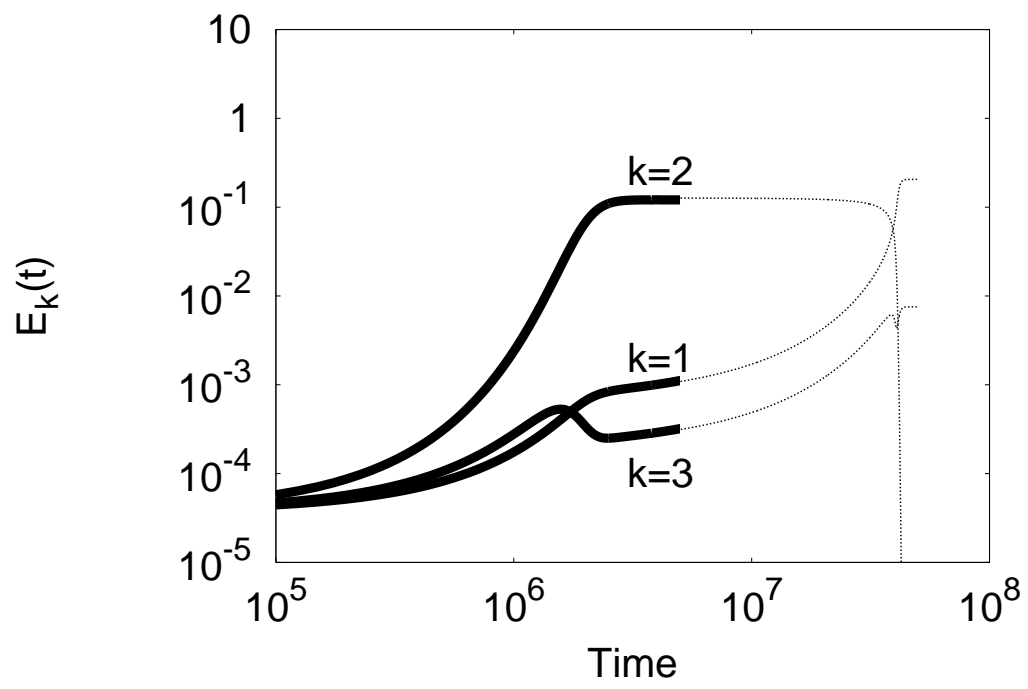

Figure 9. The comparison between DNS simulations of the Oldroyd-B model and the coarse-grained Cahn-Hilliard equation derived in the body of the text. The thicker lines represent the evolution of the lowest-energy modes in a DNS simulation, while the thinner lines are the result of a $\mathrm{CH}$ simulation. Its dynamical parameters have been set with the results obtained in section 4 This particular figure refers to a simulation with $D e=1.4, \beta=0.769$ and $R e / R e_{c}-1=0.14$.

\section{Clues on drag reduction}

One of the most striking properties of viscoleastic fluids is the drag reduction effect. In 1949, Toms found that the injection of minute amounts of polymers in turbulent fluids flowing in a channel was able to increase the mean flow by an amount soaring up to $80 \%$. Even if this phenomenon has been known for over fifty years (Toms 1949; Lumlev 1969; Virk 1975), a satisfactory understanding of its fundamental mechanisms is still lacking.

A large number of experiments has been performed to study this effect (see, e.g. Virk 1975; Nadolink \& Haigh 1995; Sreenivasan \& White 2000), but a burst in its theoretical analysis occured after drag reduction was found in numerical simulations of viscoelastic fluids (Sureshkumar et al. 1997). The activity is being spurred both by fundamental interest and industrial applications (Larson 1992).

Drag reduction is commonly associated to channel flows and boundary effects. Still, it 


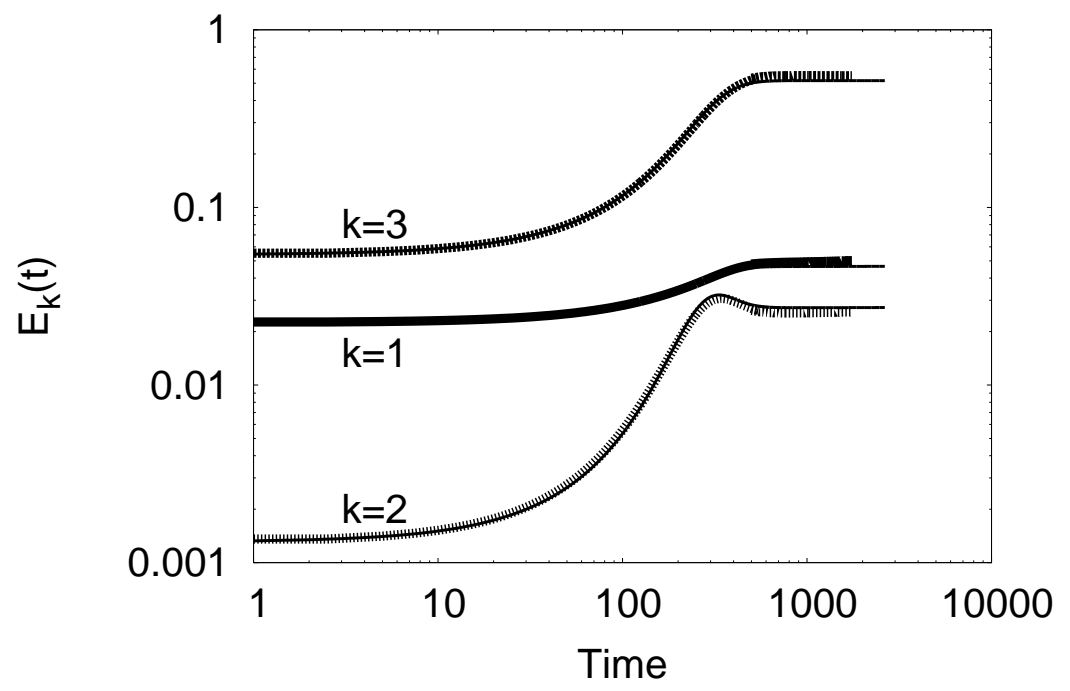

Figure 10. The generalized Cahn-Hilliard equation reproduces the dynamics of the Oldroyd-B model around the critical point $P^{*}$. As in figure 9 the thicker lines are DNS simulations while the thinner ones are $\mathrm{CH}$ simulations. This comparison was realized for $D e=1.62$ and $R e=2.5159$.

is now clear that the phenomenon is present even for free flows (Boffetta et al. 2005b). What we show here is that, even at relatively small Reynolds numbers, an increase in the Deborah number produces an enhancement in the mean flow amplitude. Simply looking at the linear stability diagram (1) we may already conclude that, as the polymer elasticity grows, so does the critical Reynolds number and the flow is stabilized. Let us further investigate this effect analytically using the results of $\S$ 团

A parameter that can be employed to study the mean flow properties in free flows is the drag coefficient $f$ (Boffetta et al. 2005b):

$$
f=\frac{F_{0} L}{U^{2}} .
$$

The drag coefficient can be seen as the ratio between the energy input (through the forcing $F_{0}$ ) and the mean energy of the flow. As we are interested in mean effects only, we will average $U^{2}$ over the basic flow periodicity. This will ensure that only mean effects will be taken into account.

When the state is linearly stable (low Reynolds numbers) we know that no perturbation can alter the basic flow, $U=V=F_{0} L^{2} / \nu$ and thus $f=R e^{-1}$.

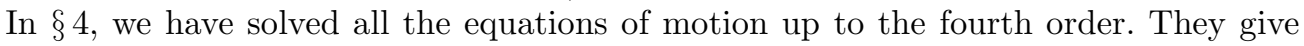
the following form of the flow (up to the second order):

$$
\begin{array}{r}
U_{x}(z)=V \cos \left(\frac{z}{L}\right)+\frac{V\left(L^{2}+(\beta-1) \nu \tau\right)}{\nu L}\left\langle w_{z}^{(1)}\right\rangle \sin \left(\frac{z}{L}\right)+ \\
-\frac{D e\left[L^{4}+\nu \tau(\beta-1)\left(2 L^{2}+\nu \tau \beta\right)\right]}{\nu^{2} \tau L}\left\langle w_{z}^{(1)}\right\rangle^{2} \cos \left(\frac{z}{L}\right)+ \\
+\frac{D e L(\beta-1)}{2}\left(\tilde{\partial}_{x}\left\langle w_{z}^{(1)}\right\rangle\right) \sin \left(\frac{2 z}{L}\right) .
\end{array}
$$

The first term is the basic, stationary Kolmogorov flow. Averaging over all possible initial conditions, component proportional to $\sin (z / L)$ and $\sin (2 z / L)$ disappear. The resulting 


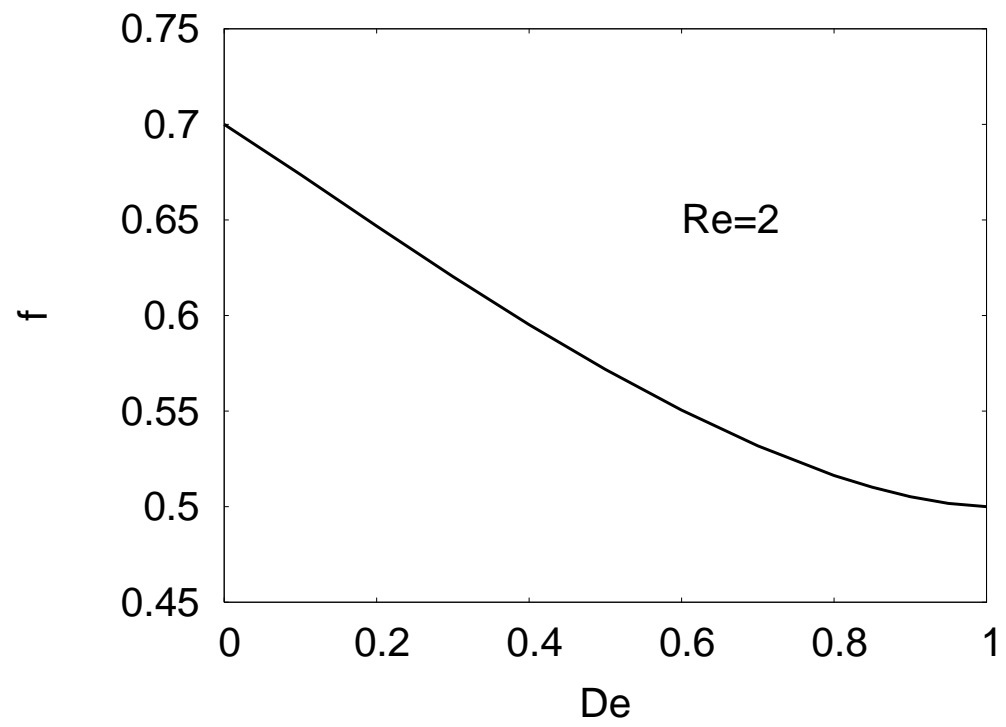

Figure 11. The drag coefficient $f$ plotted versus the Deborah number $D e$ at constant Re. As the polymer elasticity grows, the drag coefficient diminishes. This implies that the mean flow grows with $D e$.

expression for the mean flow in the $x$ direction reads:

$$
\overline{U_{x}(z)}=\left(V+h(D e, \beta) \frac{\overline{\left\langle w_{z}^{(1)}\right\rangle^{2}}}{V}\right) \cos \left(\frac{z}{L}\right)=V_{\text {eff }} \cos \left(\frac{z}{L}\right),
$$

where the quantity $\overline{\left\langle w_{z}^{(1)}\right\rangle^{2}}$ follows from the Cahn-Hilliard equation in the stationary state:

$$
0=-\partial_{x}^{2} \overline{\left\langle w_{z}^{(1)}\right\rangle} A \epsilon^{2}+\frac{B}{3} \partial_{x}^{2} \overline{\left\langle w_{z}^{(1)}\right\rangle^{3}}-\partial_{x}^{4} \overline{\left\langle w_{z}^{(1)}\right\rangle} C
$$

As $\overline{\left\langle w_{z}^{(1)}\right\rangle}$ is periodic, we can integrate twice over the domain and notice that, on the plateau, the last term is zero. The field amplitude must then satisfy:

$$
0=-A \epsilon^{2}+\frac{B}{3} \overline{\left\langle w_{z}^{(1)}\right\rangle^{2}} \Rightarrow \overline{\left\langle w_{z}^{(1)}\right\rangle}=\sqrt{\frac{3 \epsilon^{2} A}{B}}
$$

Since the analytical expression of $A$ and $B$ is known, as well as how $\epsilon$ changes with $D e$ for a fixed Reynolds number, the analytical expression for $f$ is obtained:

$$
f=\frac{\nu F L}{V_{e f f}^{2}}=\frac{V^{2}}{R e V_{e f f}^{2}}=\frac{1}{R e\left(1+h \frac{3 A}{B V^{2}} \frac{R e-R e_{c}}{R e_{c}}\right)^{2}},
$$

where $h, A, B$ and $R e_{c}$ are explicit functions of the Deborah number and $\beta$.

As we want to investigate how the polymer elasticity affects the flow, a meaningful approach is to keep the Reynolds number fixed, while varying the Deborah number. This allows studying how the same flow reacts when different kinds of polymers are injected. Once $\beta$ and $R e$ are chosen, it is possible to plot $f$ versus $D e$ on the basis of analytical results, as in figure 11 The drag coefficient is clearly decreasing with the Deborah number even though the flow is barely in its nonlinear regime.

Numerical simulations have been performed to check the consistency of these results 

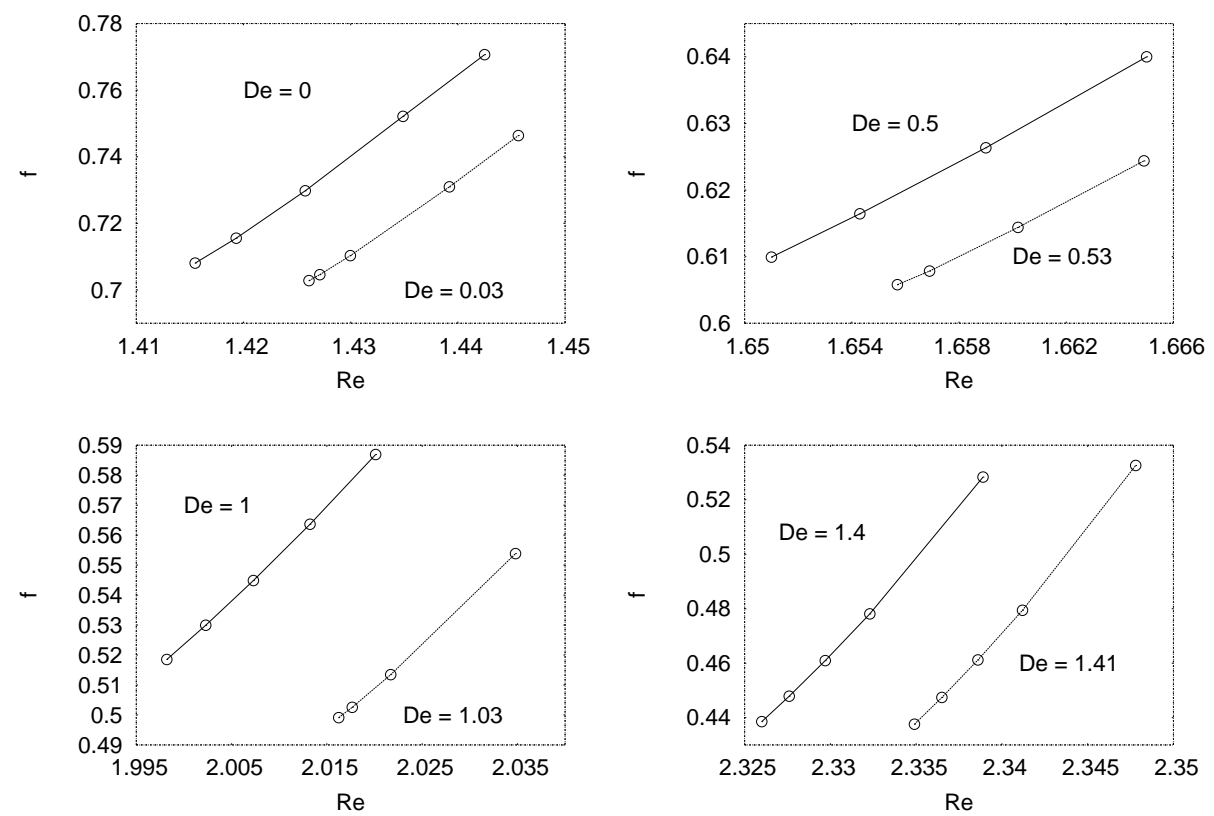

Figure 12. $\mathrm{CH}$ simulations at fixed De. the drag coefficient $f$ is found to increase with the Reynolds number. The comparison between the various curves shows that the drag coefficient reduces with the Deborah number.

and their outcome is summarized in figure 12. Here, the Deborah number has been fixed at different values and the drag coefficient has been plotted versus the Reynolds number. While $f$ increases with $R e$, as expected, larger Deborah numbers are always found to be associated to smaller drag coefficients.

\section{Conclusions}

The weakly nonlinear dynamics of a viscoelastic Kolmogorov flow has been analysed both analytically and numerically. The physical reasons for considering this flow are that, despite the fundamental difference consisting in the absence of material boundaries, it has several analogies with channel flows and is one of the few well-known exact solutions of the Oldroyd-B model.

The linear stability analysis for the Kolmogorov flow had already been developed by Boffetta et al. (2005a). No insights had however been given for the weakly nonlinear stage of evolution. This regime amounts to considering values of the Reynolds number close to the marginal stability curve separating stable from unstable regions of the phase-space. In the general nonlinear case (i.e. for arbitrarily large distances from the marginal curve), there is no way to solve the fully nonlinear equations. Conversely, close to the marginal curve, asymptotic perturbation techniques can be employed to capture the weakly nonlinear dynamics.

We found that the weakly nonlinear dynamics is described by Cahn-Hilliard-like equations, with coefficients dependent on the Deborah number. The behaviour of these coefficients with respect to $D e$ reveals that there exists a critical value of the Deborah number, where the system bifurcates to another regime. The resulting nonlinear equation still has a Cahn-Hilliard form, but contains a novel, fifth-order nonlinearity.

Above the critical $D e$, the "hydrodynamic" kink-antikink structures are replaced by 
generalized structures. We have shown that their processes of annihilation are slowed down with respect to the standard Cahn-Hilliard equation. We also found a purely nonlinear, subcritical mechanism of instability, which occurs for sufficiently large amplitudes of the initial perturbation.

Our results demonstrate that, for hydrodynamical systems governed by a standard Cahn-Hilliard equation, the presence of an additional parameter might lead to higherorder nonlinear dynamics. A system where similar phenomena are to be expected is the stratified Kolmogorov flow investigated by Balmforth \& Young (2005), with the role of elasticity played by stratification.

Our results have been obtained both exploiting the multiscale expansion and via direct numerical simulations of the original equations and their coarse-grained version. The agreement between the Cahn-Hilliard dynamics and the full-resolved one is excellent even at large times. This is true for both the standard Cahn-Hilliard and the generalized one. The asymptotic analysis is thus able to capture all of the relevant features of the flow.

In the last part of the work, we have presented some consequences for the problem of drag reduction. Although it is not common to talk about this effect in non-turbulent flows, we have shown that, even in the weakly nonlinear case, the injection of polymers induces a reduction in the drag coefficient, via the stabilization of the basic flow. Using the results of the nonlinear analysis, we have been able to give an analytical expression for the flow enhancement due to the polymers. The main qualitative conclusion is that drag reduction stems from a stabilization of the flow and appears to be a phenomenon coupling large and small scales.

\section{Acknowledgments}

This work has been supported by the Italian MIUR COFIN 2005 project n. 2005027808 (GB,AM), by Fondazione CRT-Progetto Lagrange (AB) and by the European Networks "Stirring and Mixing" HPRN-CT2002-00300 (AC) and "Non-ideal turbulence" HPRNCT-2000-00162 (MV). 


\section{Appendix A. Squire's theorem for Oldroyd-B}

Consider a parallel flow $\boldsymbol{U}=(U(z), 0)$. To investigate its stability properties one writes the linearized, nondimensional equations

$$
\begin{array}{r}
\partial_{t} \boldsymbol{w}+(\boldsymbol{u} \cdot \boldsymbol{\nabla}) \boldsymbol{w}+(\boldsymbol{w} \cdot \boldsymbol{\nabla}) \boldsymbol{u}=-\nabla q+\beta R e^{-1} \Delta \boldsymbol{w}+ \\
+(1-\beta) R e^{-1} D e^{-1} \boldsymbol{\nabla} \cdot \boldsymbol{\zeta} \\
\partial_{t} \boldsymbol{\zeta}+(\boldsymbol{u} \cdot \boldsymbol{\nabla}) \boldsymbol{\zeta}+(\boldsymbol{w} \cdot \boldsymbol{\nabla}) \boldsymbol{\sigma}=(\boldsymbol{\nabla} \boldsymbol{u})^{T} \cdot \boldsymbol{\zeta}+(\boldsymbol{\nabla} \boldsymbol{w})^{T} \cdot \boldsymbol{\sigma}+ \\
+\boldsymbol{\zeta} \cdot(\boldsymbol{\nabla} \boldsymbol{u})+\boldsymbol{\sigma} \cdot(\boldsymbol{\nabla} \boldsymbol{w})-D e^{-1} \boldsymbol{\zeta}
\end{array}
$$

where $\boldsymbol{w}$ is the perturbation of the basic profile $\boldsymbol{u}$, and $\boldsymbol{\zeta}$ is the perturbation of the basic stress tensor $\sigma$.

We now perform a Fourier transform in the directions $x$ and $y$, and in time,

$$
\begin{aligned}
w_{i}(x, y, z, t) & =\int d \omega d k_{x} d k_{y} e^{-i \omega t+k_{x} x+k_{y} y} \hat{w}_{i}\left(k_{x}, k_{y}, \omega, z\right) \\
\zeta_{i j}(x, y, z, t) & =\int d \omega d k_{x} d k_{y} e^{-i \omega t+k_{x} x+k_{y} y} \hat{\zeta}_{i j}\left(k_{x}, k_{y}, \omega, z\right)
\end{aligned}
$$

Introducing the notation

$$
\begin{gathered}
\mathrm{k}=\left(\begin{array}{c}
k_{x} \\
k_{y}
\end{array}\right) \quad \mathrm{u}=\left(\begin{array}{c}
U(z) \\
0
\end{array}\right) \quad \hat{\mathrm{w}}=\left(\begin{array}{c}
\hat{w}_{x} \\
\hat{w}_{y}
\end{array}\right) \\
\hat{\mathrm{t}}=\left(\begin{array}{c}
\hat{\zeta}_{x z} \\
\hat{\zeta}_{y z}
\end{array}\right) \quad \hat{\mathrm{z}}=\left(\begin{array}{ll}
\hat{\zeta}_{x x} & \hat{\zeta}_{x y} \\
\hat{\zeta}_{y x} & \hat{\zeta}_{y y}
\end{array}\right) \quad \mathrm{r}=\left(\begin{array}{c}
\sigma_{x z} \\
\sigma_{y z}
\end{array}\right) \quad \mathrm{s}=\left(\begin{array}{ll}
\sigma_{x x} & \sigma_{x y} \\
\sigma_{y x} & \sigma_{y y}
\end{array}\right)
\end{gathered}
$$

the linearized equations in normal modes take the form

$$
\begin{gathered}
\left(-i \omega+i \mathrm{k}^{T} \cdot \mathrm{u}\right) \hat{\mathrm{w}}+\hat{w}_{z} \frac{d \mathrm{u}}{d z}=-i \mathrm{k} \hat{q}+\beta R e^{-1}\left(-\mathrm{k}^{2}+\frac{d^{2}}{d z^{2}}\right) \hat{\mathrm{w}}+ \\
+(1-\beta) R e^{-1} D e^{-1}\left(i \hat{\mathrm{z}}^{T} \cdot \mathrm{k}+\frac{d}{d z} \hat{\mathrm{t}}\right) \\
\left(-i \omega+i \mathrm{k}^{T} \cdot \mathrm{u}\right) \hat{w}_{z}=-\frac{d \hat{q}}{d z}+\beta R e^{-1}\left(-\mathrm{k}^{2}+\frac{d^{2}}{d z^{2}}\right) \hat{w}_{z}+ \\
+(1-\beta) R e^{-1} D e^{-1}\left(i \mathrm{k}^{T} \cdot \hat{\mathrm{t}}+\frac{d}{d z} \hat{\zeta}_{z z}\right) \\
\left(-i \omega+i \mathrm{k}^{T} \cdot \mathrm{u}+D e^{-1}\right) \hat{z}+\hat{w}_{z} \frac{d}{d z} \mathrm{~s}=\hat{\mathrm{t}} \cdot \frac{d \mathrm{u}}{d z}+\frac{d \mathrm{u}}{d z} \cdot \hat{\mathrm{t}}^{T}+ \\
+i(\mathrm{~s} \cdot \mathrm{k}) \hat{\mathrm{w}}^{T}+i \hat{\mathrm{w}}\left(\mathrm{k}^{T} \cdot \mathrm{s}\right)+\mathrm{r} \frac{d}{d z} \hat{\mathrm{w}}^{T}+\frac{d \hat{\mathrm{w}}^{T}}{d z} \mathrm{r}^{T} \\
\left(-i \omega+i \mathrm{k}^{T} \cdot \mathrm{u}+D e^{-1}\right) \hat{\mathrm{t}}+\hat{w}_{z} \frac{d}{d z} \mathrm{r}=\hat{\zeta}_{z z} \frac{d \mathrm{u}}{d z}+ \\
+i(\mathrm{~s} \cdot \mathrm{k}) \hat{w}_{z}+i \hat{\mathrm{w}}\left(\mathrm{r}^{T} \cdot \mathrm{k}\right)+\mathrm{r} \frac{d}{d z} \hat{w}_{z}+\frac{d}{d z} \hat{\mathrm{w}} \\
\left(-i \omega+i \mathrm{k}^{T} \cdot \mathrm{u}+D e^{-1}\right) \hat{\zeta}_{z z}=2 i\left(\mathrm{r}^{T} \cdot \mathrm{k}\right) \hat{w}_{z}+2 \frac{d}{d z} \hat{w}_{z}
\end{gathered}
$$


24 A. Bistagnino, G. Boffetta, A. Celani, A. Mazzino, A. Puliafito, M. Vergassola

Consider the following transformation

$$
\begin{gathered}
\bar{k}_{x}=|\mathrm{k}| \quad \bar{w}_{x}=\frac{\mathrm{k}^{T} \cdot \hat{\mathrm{w}}}{|\mathrm{k}|} \quad \bar{w}_{z}=\hat{w}_{z} \quad \bar{q}=\frac{|\mathrm{k}|}{k_{x}} \hat{q} \\
\overline{R e}=\frac{k_{x}}{|\mathrm{k}|} \operatorname{Re} \quad \overline{D e}=\frac{k_{x}}{|\mathrm{k}|} D e \quad \bar{\omega}=\frac{|\mathrm{k}|}{k_{x}} \omega \\
\bar{t}_{x}=\frac{k_{x}}{|\mathrm{k}|} \frac{\mathrm{k}^{T} \cdot \hat{\mathrm{t}}}{|\mathrm{k}|} \bar{\zeta}_{x x}=\frac{k_{x}}{|\mathrm{k}|} \frac{\mathrm{k}^{T} \cdot \hat{z} \cdot \mathrm{k}}{|\mathrm{k}|^{2}} \quad \bar{\zeta}_{z z}=\frac{k_{x}}{|\mathrm{k}|} \hat{\zeta}_{z z}
\end{gathered}
$$

From (A 6)- (A 10) one can derive the equations for the overlined variables

$$
\begin{gathered}
{\left[-i \bar{\omega}+i \bar{k}_{x} U(z)\right] \bar{w}_{x}+\bar{w}_{z} \frac{d U}{d z}=-i \bar{k}_{x} \bar{q}+\beta \overline{R e}^{-1}\left(-\bar{k}_{x}^{2}+\frac{d^{2}}{d z^{2}}\right) \bar{w}_{x}+} \\
+(1-\beta) \overline{R e}^{-1} \overline{D e}^{-1}\left(i \bar{k}_{x} \bar{\zeta}_{x x}+\frac{d}{d z} \bar{t}_{x}\right) \\
{\left[-i \bar{\omega}+i \bar{k}_{x} U(z)\right] \bar{w}_{z}=-\frac{d \hat{q}}{d z}+\beta \overline{R e}^{-1}\left(-\bar{k}_{x}^{2}+\frac{d^{2}}{d z^{2}}\right) \bar{w}_{z}+} \\
+(1-\beta) \overline{R e}^{-1} \overline{D e}^{-1}\left(i \bar{k}_{x} \bar{t}_{x}+\frac{d}{d z} \bar{\zeta}_{z z}\right) \\
{\left[-i \bar{\omega}+i \bar{k}_{x} U(z)+\overline{D e}^{-1}\right] \bar{\zeta}_{x x}+\bar{w}_{z} \frac{d \bar{s}_{x x}}{d z}=2 \bar{t}_{x} \frac{d U}{d z}+2 i \bar{k}_{x} \bar{s}_{x x} \bar{w}_{x}+2 \bar{r}_{x} \frac{d \bar{w}_{x}}{d z}} \\
{\left[-i \bar{\omega}+i \bar{k}_{x} U(z)+\overline{D e}^{-1}\right] \bar{t}_{x}+\bar{w}_{z} \frac{d}{d z} \bar{r}_{x}=\bar{\zeta}_{z z} \frac{d U}{d z}+i \bar{s}_{x x} \bar{k}_{x} \bar{w}_{z}+i \bar{k}_{x} \bar{w}_{x} \bar{r}_{x}} \\
+\bar{r}_{x} \frac{d \bar{w}_{z}}{d z}+\frac{d \bar{w}_{x}}{d z} \\
{\left[-i \bar{\omega}+i \bar{k}_{x} U(z)+\overline{D e}^{-1}\right] \bar{\zeta}_{z z}=2 i \bar{k}_{x} \bar{r}_{x} \bar{w}_{z}+2 \frac{d \bar{w}_{z}}{d z}}
\end{gathered}
$$

where we introduced the quantities

$$
\bar{s}_{x x}=\frac{\mathrm{k}^{T} \cdot \mathrm{s} \cdot \mathrm{k}}{|\mathrm{k}|^{2}}=1+\overline{D e}^{2}\left[U^{\prime}(z)\right]^{2}, \bar{r}_{x}=\frac{\mathrm{k}^{T} \cdot \mathrm{r}}{|\mathrm{k}|}=\overline{D e}^{2} U^{\prime}(z)
$$

Equations (A 12)-A 16) are exactly the same as A 6 - A 10 but with $k_{y}=0, \hat{w}_{y}=$ $0, \zeta_{x y}=\zeta_{y y}=\zeta_{y z}=0$. Therefore they describe a two-dimensional linear disturbance of the basic flow at smaller Reynolds and Deborah numbers. If the three-dimensional perturbation $\boldsymbol{w}, \boldsymbol{\zeta}$ is unstable at $(R e, D e)$, then the two-dimensional disturbance $\overline{\boldsymbol{w}}, \overline{\boldsymbol{\zeta}}$ is unstable at $(\overline{R e}, \overline{D e})$ and its rate of growth is larger $(\operatorname{Im}(\bar{\omega}) \geq \operatorname{Im}(\omega)>0)$.

\section{Appendix B. Painlevé analysis}

We perform a Painlevé analysis to ascertain whether the fifth-order equation (5.1) is integrable as the usual cubic Cahn-Hilliard equation (4.6).

After rescaling dependent and independent variables, the stationary equation takes the form:

$$
-u-\frac{u^{3}}{3}-\lambda \partial_{x}^{2} u+\frac{\gamma}{5} u^{5}=0
$$

The Painlevé test consists in checking whether the structure of the solution around singularities in the complex plane has the form of a Laurent series. A simple balance of 
the last two terms in the equation indicates that the singularity has order $-1 / 2$. The putative Laurent series should then be sought as:

$$
u(z)=z^{-1 / 2}\left[u_{0}+u_{1} z+u_{2} z^{2}+u_{3} z^{3}+\cdots\right],
$$

where $z$ is the complex variable denoting the separation from the singularity $z_{*}$. When the series (B2) is inserted into equation (B1), a hierarchy of equations of the form

$a_{k} u_{k}=b_{k}$ is obtained. The $a_{k}$ 's and $b_{k}$ 's can be calculated in terms of $u_{k-1}, \cdots, u_{0}$. The impossibility for an arbitrary equation to have a Laurent series expansion is due to resonances, i.e.values of $k$ such that $a_{k}=0$. Integrability is equivalent to checking that $b_{k}=0$ for the orders corresponding to resonances. In our case, it is easy to check that

$$
a_{k}=-\lambda\left(k-\frac{1}{2}\right)\left(k-\frac{3}{2}\right)+\gamma u_{0}^{4} ; \quad u_{0}=\left(\frac{15 \lambda}{4 \gamma}\right)^{1 / 4} \mapsto a_{k}=-\lambda(k+1)(k-3) .
$$

The resonance is therefore at the third order and we need to perform the explicit calculation up to that order to check whether or not $b_{3}=0$. The algebra is elementary and the coefficients are:

$$
u_{1}=\frac{u_{0}^{3}}{12 \lambda}, u_{2}=\frac{u_{0}}{\lambda}\left[\frac{1}{3}+\frac{5}{128 \gamma}\right] .
$$

Using these values, one can verify that

$$
b_{3}=2 \gamma u_{0}^{2} u_{1}^{3}+4 \gamma u_{0}^{3} u_{1} u_{2}-u_{1}-u_{0}^{2} u_{2}-u_{0} u_{1}^{2}
$$

vanishes and the Painlevé test is satisfied.

\section{REFERENCES}

Ablowitz, M.J. \& Clarkson, P.A. 1991 Solitons, Nonlinear Evolution Equations and Inverse Scattering. Cambridge University Press.

Arnold, V.I. \& Meshalkin, L. 1960 A.N. Kolmogorov's seminar on selected problems of analysis (1958-1959). Uspekhi Mat. Nauk 15, 247-250.

Balmforth, N.J., Young, Y.-N. 2995 Stratified Kolmogorov flow. Part 2. J. Fluid Mech. 528, 23-42.

Bayly, B.J., Orszag, S.A., Herber,T. 1988 Instability mechanisms in shear-flow transition. Annu. Rev. Fluid Mech. 20, 359-391.

Bensoussan, A., Lions, J.-L. \& Papanicolau, G. 1978 Asymptotic Analysis for Periodic Structures. North-Holland.

Bird, R.B., Hassager, O., Armstrong, R.C. \& Curtiss, C.F. 1987 Dynamics of Polymeric Liquids. Wiley-Interscience.

Boffetta, G., Celani, A., Mazzino, A., Puliafito, A. \& Vergassola, M. 2005a The viscoelastic Kolmogorov flow: eddy viscosity and linear stability. J. Fluid Mech. 523, 161170.

Boffetta, G., Celani, A. \& Mazzino, A. 2005b Drag reduction in the turbulent Kolmogorov flow. Phys. Rev. E 71, 036307.

Bray, A.J. 2002 Theory of phase-ordering kinetics. Advances in Physics 51, 481587

Cahn, J.W. \& Hilliard, J.E. 1958 Free energy of a non uniform system. J. Chem. Phys. 28, 258.

Canuto, C., Hussaini, M.Y., Quarteroni, A. \& Zang, T. A. 1988 Spectral Methods in Fluid Dynamics. Springer-Verlag.

Gama, S., Vergassola, M. \& Frisch, U. 1994 Negative eddy-viscosity in isotropically forced two-dimensional flow: linear and nonlinear dynamics. J. Fluid Mech. 260, 95-126.

Geraschenko, S., Chevallard, C. \& Steinberg, V. 2005 Single-polymer dynamics: Coilstretch transition in a random flow. Europhys. Lett. 71(2), 221-227.

Hinch, E.J. 1977 Mechanical models of dilute polymer solutions in strong flows. Phys. Fluids 20, S22-S30. 
KaWAsaki, K. \& OHta, T 1982 Kink dynamics in one-dimensional nonlinear systems. Physica A 116573.

Karttunen, M., Vattulainen, I. \& Lukkarinen, A. 2004 Novel Methods in Soft Matter Simulations. Springer-Verlag.

Khouider, B., Majda, A.J. \& Katsoulakis, M.A. 2003 Coarse-grained stochastic models for tropical convection and climate. Proc. Natl. Acad. Sci 100, 1194111946.

Kowalesvki, S. 1889 Sur le problème de la rotation d'un corps solide autur d'un point fixe. Acta Math. 12, 177-232.

Kowalesvki, S. 1890 Sur une propriété du système d'équations différentielles qui définit la rotation d'un corpe solide autour d'un point fixe. Acta Math. 14, 81-93.

Larson, R.G. 1992 Instabilities in viscoelastic flows. Rheol. Acta 31, 213-263.

Legras, B. \& Villone, B. 2003 Dispersive and friction-induced stabilization of the CahnHilliard inverse cascade. Physica D 175, 139-166.

Lumley, J. 1969 Drag reduction by additives. Annu. Rev. Fluid Mech. 1, 367-384.

Manfroi, A., \& Young, W. 1999 Slow evolution of zonal jets on the beta-plane. J. Atmos. Science 56, 784-800.

Meshalkin, L. \& Sinai, Ya G. 1961 Investigation of the stability of a stationary solution of a system of equations for the plane movement of an incompressible viscous fluid. J. Appl. Math. Mech. 25, 1700-1705.

NAdolink, R.H. \& Haigh, W.W. 1997 Bibliography on skin friction reduction with polymers and other boundary-layer additives. ASME Appl. Mech. Rev. 48, 351-460.

Nepomnyashchyi, A.A. 1976 On the stability of the secondary flow of a viscous fluid in an infinite domain. Appl. Math. Mech. 40, 886-891.

Oldroyd, J.G. 1950 On the formulation of rheological equations of state. Proc. Roy. Soc. London Ser A 200, 523-541.

Painlevé, P. 1897 Leçons sur la Théorie Analytique des Équations Differentielles. Hermann, Paris.

Pedlosky, J. 1987 Geophysical fluid dynamics 2nd edition. Springer.

She, Z.S. 1987 Metastability and vortex pairing in the Kolmogorov flow. Phys. Lett. A 124, 161-164.

Sivashinsky, G.I. 1985 Weak turbulence in periodic flows. Physica D 17, 243-255.

Sreenivasan, K.R. \& White, C.M. 2000 The onset of drag reduction by dilute polymer additives, and the maximum drag reduction asymptote. J. Fluid Mech. 409, 149-164.

Sureshrumar, R., Beris, A.N., \& HAndler, R.A. 1997 Direct numerical simulation of polymer-induced drag reduction in turbulent channel flow. Phys. Fluids 9, 743-755.

Toms, B.A. 1949 Observation on the flow of linear polymer solutions through straight tubes at large Reynolds numbers. Proc. 1st International Congress on Rheology 2, 135-141.

Vattulainen, I. \& Karttunen, M. 2005 Computational Nanotechnology, edited by M. Rieth and W. Schommers. American Scientific Press, in press.

VIRK, P. S. 1975 Drag reduction fundamentals. AIChE Journal 21, 625-656. 